\title{
Sobolev boundedness and continuity for commutators of the local Hardy-Littlewood maximal function
}

\author{
Feng LiU, QINGYing XUE* and Kôzô Yabuta
}

\begin{abstract}
Let $\Omega$ be a subdomain in $\mathbb{R}^{n}$ and $M_{\Omega}$ be the local Hardy-Littlewood maximal function. In this paper, we show that both the commutator and the maximal commutator of $M_{\Omega}$ are bounded and continuous from the first order Sobolev spaces $W^{1, p_{1}}(\Omega)$ to $W^{1, p}(\Omega)$ provided that $b \in W^{1, p_{2}}(\Omega), 1<p_{1}, p_{2}, p<\infty$ and $1 / p=1 / p_{1}+1 / p_{2}$. These are done by establishing several new pointwise estimates for the weak derivatives of the above commutators. As applications, the bounds of these operators on the Sobolev space with zero boundary values are obtained.

\section{Paikallisen Hardyn-Littlewoodin maksimaalifunktion kommutaattoreiden rajoittuneisuus ja jatkuvuus Sobolevin avaruuksissa}

Tiivistelmä. Olkoon $\Omega$ avaruuden $\mathbb{R}^{n}$ alue ja $M_{\Omega}$ paikallinen Hardyn-Littlewoodin maksimaalifunktio. Tässä työssä osoitamme, että sekä operaattorin $M_{\Omega}$ kommutaattori että sen maksimaalinen kommutaattori ovat rajoitettuja ja jatkuvia ensimmäisen kertaluvun Sobolevin avaruudesta $W^{1, p_{1}}(\Omega)$ avaruuteen $W^{1, p}(\Omega)$, mikäli $b \in W^{1, p_{2}}(\Omega), 1<p_{1}, p_{2}, p<\infty$ ja $1 / p=1 / p_{1}+1 / p_{2}$. Nämä tulokset seuraavat em. kommutaattorien heikkoja derivaattoja koskevista uusista pisteittäisistä arvioista, joita todistamme useita. Sovelluksina saamme nämä operaattorit rajoitetuiksi nollareuna-arvoisissa Sobolevin avaruuksissa.
\end{abstract}

\section{Introduction}

1.1. Background. Let $\Omega$ be a subdomain in $\mathbb{R}^{n}$ and $\Omega^{c}=\mathbb{R}^{n} \backslash \Omega$. Let $f$ be a measurable function defined from the subdomain $\Omega$ to $\mathbb{R}$. The local HardyLittlewood maximal operator $M_{\Omega}$ is defined by

$$
M_{\Omega} f(x)=\sup _{0<r<\operatorname{dist}\left(x, \Omega^{c}\right)} \frac{1}{|B(x, r)|} \int_{B(x, r)}|f(y)| d y,
$$

where $B(x, r)$ is a ball in $\mathbb{R}^{n}$ centered at $x$ with radius $r$. When $\Omega=\mathbb{R}^{n}$, the operator $M_{\Omega}$ coincides with the classical centered Hardy-Littlewood maximal operator $M$. It was well known that $M$ is $L^{p}\left(\mathbb{R}^{n}\right)$ bounded for $1<p \leq \infty$, and is bounded from $L^{1}\left(\mathbb{R}^{n}\right)$ to $L^{1, \infty}\left(\mathbb{R}^{n}\right)$. A simple observation may yield that $M_{\Omega} f(x) \leq M\left(f \chi_{\Omega}\right)(x)$ for all $x \in \Omega$. Therefore, $M_{\Omega}$ is also bounded on $L^{p}(\Omega)$ for $1<p \leq \infty$ and is bounded from $L^{1}(\Omega)$ to $L^{1, \infty}(\Omega)$.

The regularity theory of maximal operators has been the subject of many recent articles in Harmonic analysis. The first work was due to Kinnunen [16] who proved

https://doi.org/10.54330/afm.113296

2020 Mathematics Subject Classification: Primary 42B25, 46E35.

Key words: Commutator, maximal commutator, local Hardy-Littlewood maximal function, Sobolev space, boundedness, continuity.

The first author was supported partly by NSFC (Grant No. 11701333) and SP-OYSTTTCMSS (Grant No. Sxy2016K01). The second author was partly supported by the National Key R\&D Program of China (No. 2020YFA0712900), NSFC (No. 11871101) and 111 Project.

* Corresponding author.

(c) 2022 The Finnish Mathematical Society 
that $M$ is bounded on the first order Sobolev space $W^{1, p}\left(\mathbb{R}^{n}\right)$ for $1<p \leq \infty$. Later on, Kinnunen's result was extended to various cases. For example, see [17] for the local case, [19] for the fractional case, $[8,21]$ for the multisublinear case. It is well known that $M$ is continuous on $L^{p}\left(\mathbb{R}^{n}\right)$ for all $1<p \leq \infty$, which follows directly from the well-known $L^{p}$ bounds and the sublinearity. However, the continuity of $M: W^{1, p}\left(\mathbb{R}^{n}\right) \rightarrow W^{1, p}\left(\mathbb{R}^{n}\right)$ for $1<p<\infty$ is certainly a nontrivial issue, since the maximal operator is not necessarily sublinear at the derivative level. This continuity property was investigated by Luiro [25] and later extensions were given in [26, 8]. More interesting works related to this topic may be found in $[2,6,7,9,20,22,23]$, see also the nice recent survey paper given by Carneiro in [5].

In the global case, Sobolev regularity results can even be extended to the situation of sublinear operators that commute with translations [12]. It should be pointed out that the methods of dealing the Sobolev regularity for maximal operators in global case $\Omega=\mathbb{R}^{n}$ and the local case $\Omega \subsetneq \mathbb{R}^{n}$ are quite different. An important reason is that the local maximal operator $M_{\Omega}$ lacks the commutativity with translations, which plays a key role in the study of the $W^{1, p}$-bounds for $M$. The first result addressing the local $\Omega \subsetneq \mathbb{R}^{n}$ theory was given by Kinnunen and Lindqvist [17] who proved that the map $M_{\Omega}: W^{1, p}(\Omega) \rightarrow W^{1, p}(\Omega)$ is bounded for all $1<p \leq \infty$, where $W^{1, p}(\Omega)$ is the first order Sobolev space as follows:

$$
W^{1, p}(\Omega):=\left\{f: \Omega \rightarrow \mathbb{R}:\|f\|_{1, p, \Omega}=\|f\|_{p, \Omega}+\|\nabla f\|_{p, \Omega}<\infty\right\},
$$

where $\|f\|_{p, \Omega}=\|f\|_{L^{p}(\Omega)}$ and $\nabla f=\left(D_{1} f, \ldots, D_{n} f\right)$ is the weak gradient of $f$.

Actually, Kinnunen and Lindqvist obtained the $W^{1, p}(\Omega)$ bounds of $M_{\Omega}$ by proving the following key estimate:

$$
\left|\nabla M_{\Omega} f(x)\right| \leq 2 M_{\Omega}(\nabla f)(x)
$$

for almost every $x \in \Omega$ and $f \in W^{1, p}(\Omega)$ for some $1<p<\infty$ (also see [12]). Later on, the above result was extended to the fractional case in[14] and to the multilinear case in [13].

The main purpose of this paper is to investigate the Sobolev boundedness and continuity properties for two classes of commutators of the local Hardy-Littlewood maximal function. We start with the definitions of the commutators.

Definition 1.1. Let $b$ be a locally integrable function defined on $\Omega$. The commutator of the centered Hardy-Littlewood maximal function $\left[b, M_{\Omega}\right]$ and the maximal commutator of $M_{b, \Omega}$ are defined respectively by

$$
\left[b, M_{\Omega}\right](f)(x)=b(x) M_{\Omega} f(x)-M_{\Omega}(b f)(x), \quad x \in \Omega
$$

and

$$
M_{b, \Omega} f(x)=\sup _{0<r<\operatorname{dist}\left(x, \Omega^{c}\right)} \frac{1}{|B(x, r)|} \int_{B(x, r)}|b(x)-b(y)||f(y)| d y, \quad x \in \Omega .
$$

When $\Omega=\mathbb{R}^{n}$, the operator $\left[b, M_{\Omega}\right]$ (resp., $M_{b, \Omega}$ ) coincides with the classical commutator $[b, M]$ (resp., $\left.M_{b}\right)$. Milman and Schonbek [27] first proved the $L^{p}(1<$ $p<\infty)$ bounds of $[b, M]$. The above result was improved by Bastero et al. [3] for $b \in \operatorname{BMO}\left(\mathbb{R}^{n}\right)$. Recently, Agcayazi et al. [1] established the end-point estimates for $[b, M]$. An important application is that the operator $[b, M]$ can be used in studying the product of a function in $H^{1}\left(\mathbb{R}^{n}\right)$ and a function in $\operatorname{BMO}\left(\mathbb{R}^{n}\right)$ (see [4] for instance). The boundedness of $M_{b}$ has also been studied intensively by many authors (see [1, 10, 15, 28]). Recently, the authors [24] investigated the regularity of 
commutators of the Hardy-Littlewood maximal function. More precisely, they gave the following result.

Theorem A. [24] Let $1<p_{1}, p_{2}, p<\infty$ and $1 / p=1 / p_{1}+1 / p_{2}$. If $b \in W^{1, p_{2}}\left(\mathbb{R}^{n}\right)$, then the map $[b, M]: W^{1, p_{1}}\left(\mathbb{R}^{n}\right) \rightarrow W^{1, p}\left(\mathbb{R}^{n}\right)$ is bounded and continuous. Moreover, the map $M_{b}: W^{1, p_{1}}\left(\mathbb{R}^{n}\right) \rightarrow W^{1, p}\left(\mathbb{R}^{n}\right)$ is bounded. Especially, if $f \in W^{1, p_{1}}\left(\mathbb{R}^{n}\right)$, it holds pointwisely

$$
|\nabla[b, M](f)(x)| \leq|\nabla b(x)| M f(x)+|b(x)| M|\nabla f|(x)+M(|\nabla(b f)|)(x)
$$

for almost every $x \in \mathbb{R}^{n}$.

Based on the above analysis, a natural question arises

Question 1.2. What kinds of regularity properties do the commutators $\left[b, M_{\Omega}\right]$ and $M_{b, \Omega}$ enjoy?

Question 1.2 is the main motivation of the current work. However, as it was mentioned before, these operators lack the commutativity with translations. Therefore, this question belongs to less fine questions. More obstacles must be overcome and some new pointwise estimates should be established.

Before addressing this problem, let us point out some useful facts.

- The operator $\left[b, M_{\Omega}\right]$ is neither positive nor sublinear. The map $\left[b, M_{\Omega}\right]$ : $L^{p_{1}}(\Omega) \rightarrow L^{p}(\Omega)$ is bounded and continuous if $b \in L^{p_{2}}(\Omega), 1<p_{1}, p_{2}, p \leq \infty$ and $1 / p=1 / p_{1}+1 / p_{2}$. Moreover, it holds that

$$
\|[b, M](f)\|_{p, \Omega} \leq C_{p_{1}, p_{2}, n}\|f\|_{p_{1}, \Omega}\|b\|_{p_{2}, \Omega} .
$$

- The operator $M_{b, \Omega}$ is positive and sublinear. The map $M_{b, \Omega}: L^{p_{1}}(\Omega) \rightarrow L^{p}(\Omega)$ is bounded and continuous if $b \in L^{p_{2}}(\Omega), 1<p_{1}, p_{2}, p \leq \infty$ and $1 / p=$ $1 / p_{1}+1 / p_{2}$. Moreover,

$$
\left\|M_{b, \Omega} f\right\|_{p, \Omega} \leq C_{p_{1}, p_{2}, n}\|f\|_{p_{1}, \Omega}\|b\|_{p_{2}, \Omega} .
$$

This can be seen easily from the boundedness of $M_{\Omega}$ and the fact that

$$
M_{b, \Omega} f(x) \leq|b(x)| M_{\Omega} f(x)+M_{\Omega}(b f)(x), \quad x \in \Omega .
$$

The main results in this paper are as follows:

Theorem 1.1. Let $1<p_{1}, p_{2}, p<\infty$ and $1 / p=1 / p_{1}+1 / p_{2}$. If $b \in W^{1, p_{2}}(\Omega)$, then the map $\left[b, M_{\Omega}\right]: W^{1, p_{1}}(\Omega) \rightarrow W^{1, p}(\Omega)$ is bounded and continuous. Moreover, if $f \in W^{1, p_{1}}(\Omega)$, then

$$
\begin{aligned}
\left|\nabla\left[b, M_{\Omega}\right](f)(x)\right| \leq & |\nabla b(x)|\left|M_{\Omega} f(x)\right|+2|b(x)| M_{\Omega}|\nabla f|(x) \\
& +2 M_{\Omega}(|\nabla b| f)(x)+2 M_{\Omega}(b|\nabla f|)(x),
\end{aligned}
$$

for almost every $x \in \Omega$. Consequently, it holds that

$$
\left\|\left[b, M_{\Omega}\right](f)\right\|_{1, p, \Omega} \leq C_{p_{1}, p_{2}, n}\|b\|_{1, p_{2}, \Omega}\|f\|_{1, p_{1}, \Omega} .
$$

Theorem 1.2. Assume $|\Omega|<\infty$. Let $1<p_{1}, p_{2}, p<\infty$ and $1 / p=1 / p_{1}+1 / p_{2}$. If $b \in W^{1, p_{2}}(\Omega)$, then the map $M_{b, \Omega}: W^{1, p_{1}}(\Omega) \rightarrow W^{1, p}(\Omega)$ is bounded and continuous. Moreover, if $f \in W^{1, p_{1}}(\Omega)$, then

$$
\left|\nabla M_{b, \Omega} f(x)\right| \leq 2\left(M_{b, \Omega}|\nabla f|(x)+M_{\Omega}(|\nabla b| f)(x)\right)+|\nabla b|(x) M_{\Omega} f(x),
$$

for almost every $x \in \Omega$. Consequently, it holds that

$$
\left\|M_{b, \Omega} f\right\|_{1, p, \Omega} \leq C_{p_{1}, p_{2}, n}\|b\|_{1, p_{2}, \Omega}\|f\|_{1, p_{1}, \Omega} .
$$


This paper will be organized as follows. Section 2 will be devoted to proving Theorem 1.1. In Section 3 we shall prove Theorem 1.2. Finally, we shall prove that the commutator of local Hardy-Littlewood maximal function preserves the zero boundary values in Sobolev's sense in Section 4. We would like to remark that our main proofs are motivated by the ideas in $[14,17,26]$, but our methods and techniques are more complex than those of $[14,17,26]$.

Throughout this paper, the letter $C$ will stand for positive constants not necessarily the same one at each occurrence but independent of the essential variables. Especially, the letter $C_{\alpha, \beta}$ denote the positive constants that depend on the parameters $\alpha, \beta$.

\section{Proof of Theorem 1.1}

In this section we shall present the proof of Theorem 1.1. We start with presenting the following proposition, which plays a key role in the proof of Theorem 1.2.

Proposition 2.1. $[14,17]$ Let $1 \leq p \leq \infty$. If $f_{k} \rightarrow f, g_{k} \rightarrow g$ weakly in $L^{p}(\Omega)$ and $f_{k} \leq g_{k}(k=1,2, \ldots)$ almost everywhere in $\Omega$, then $f \leq g$ almost everywhere in $\Omega$.

The following lemma is the main ingredient of proving Theorem 1.1.

Lemma 2.2. Let $1<p_{1}, p_{2}, p<\infty$ and $1 / p=1 / p_{1}+1 / p_{2}$. If $f \in W^{1, p_{1}}(\Omega)$ and $g \in W^{1, p_{2}}(\Omega)$, then $f g \in W^{1, p}(\Omega)$. Moreover,

$$
\nabla(f g)=g \nabla f+f \nabla g
$$

almost everywhere in $\Omega$. In particular, it holds that

$$
\|f g\|_{1, p, \Omega} \leq\|f\|_{1, p_{1}, \Omega}\|g\|_{1, p_{2}, \Omega}
$$

Proof. Since $f \in W^{1, p_{1}}(\Omega)$ and $g \in W^{1, p_{2}}(\Omega)$, there exist a sequence $\left\{\varphi_{j}\right\}_{j=1}^{\infty}$ of functions in $W^{1, p_{1}}(\Omega) \cap \mathcal{C}^{\infty}(\Omega)$ and a sequence $\left\{\psi_{j}\right\}_{j=1}^{\infty}$ of functions in $W^{1, p_{2}}(\Omega) \cap$ $\mathcal{C}^{\infty}(\Omega)$ such that $\varphi_{j} \rightarrow f$ in $W^{1, p_{1}}(\Omega)$ and $\psi_{j} \rightarrow g$ in $W^{1, p_{2}}(\Omega)$ as $j \rightarrow \infty$. Fix $j \in \mathbb{N}$. For all $i=1,2, \ldots, n$ and every $x \in \Omega$, by Leibniz rule

$$
D_{i}\left(\varphi_{j} \psi_{j}\right)=\left(D_{i} \varphi_{j}\right) \psi_{j}+\left(D_{i} \psi_{j}\right) \varphi_{j}
$$

By Hölder's inequality and Minkonwski's inequality, one has

$$
\begin{aligned}
& \left\|\left(D_{i} \varphi_{j}\right) \psi_{j}-\left(D_{i} f\right) g\right\|_{p, \Omega} \leq\left\|\left(D_{i} \varphi_{j}-D_{i} f\right) \psi_{j}+D_{i} f\left(\psi_{j}-g\right)\right\|_{p, \Omega} \\
& \leq\left\|D_{i} \varphi_{j}-D_{i} f\right\|_{p_{1}, \Omega}\left\|\psi_{j}\right\|_{p_{2}, \Omega}+\left\|D_{i} f\right\|_{p_{1}, \Omega}\left\|\psi_{j}-g\right\|_{p_{2}, \Omega} \\
& \leq\left\|\varphi_{j}-f\right\|_{1, p_{1}, \Omega}\left(\left\|\psi_{j}-g\right\|_{p_{2}, \Omega}+\|g\|_{p_{2}, \Omega}\right)+\left\|D_{i} f\right\|_{p_{1}, \Omega}\left\|\psi_{j}-g\right\|_{p_{2}, \Omega} .
\end{aligned}
$$

which implies that $\left(D_{i} \varphi_{j}\right) \psi_{j} \rightarrow\left(D_{i} f\right) g$ in $L^{p}(\Omega)$ as $j \rightarrow \infty$. Similarly we get $\left(D_{i} \psi_{j}\right) \varphi_{j} \rightarrow\left(D_{i} g\right) f$ in $L^{p}(\Omega)$ as $j \rightarrow \infty$. These facts together with (2.3) imply that $D_{i}\left(\varphi_{j} \psi_{j}\right) \rightarrow g\left(D_{i} f\right)+f\left(D_{i} g\right)$ in $L^{p}(\Omega)$ as $j \rightarrow \infty$. On the other hand, it is not difficult to check that $\varphi_{j} \psi_{j} \rightarrow f g$ in $L^{p}(\Omega)$ as $j \rightarrow \infty$. Therefore, by the above facts we have that for every $\phi \in \mathcal{C}_{0}^{\infty}(\Omega)$,

$$
\begin{aligned}
\int_{\Omega} f(x) g(x) D_{i} \phi(x) d x & =\lim _{j \rightarrow \infty} \int_{\Omega} \varphi_{j}(x) \psi_{j}(x) D_{i} \phi(x) d x \\
& =-\lim _{j \rightarrow \infty} \int_{\Omega} D_{i}\left(\varphi_{j} \psi_{j}\right)(x) \phi(x) \\
& =-\lim _{j \rightarrow \infty} \int_{\Omega} \phi(x)\left(g(x) D_{i} f(x)+f(x) D_{i} g(x)\right) d x
\end{aligned}
$$


which yields that $D_{i}(f g)=g D_{i} f+f D_{i} g$ almost everywhere in $\Omega$. This gives (2.1). By (2.1) and Hölder's inequality, we have

$$
\begin{aligned}
\|f g\|_{1, p, \Omega} & \leq\|f g\|_{p_{, \Omega}}+\|\nabla(f g)\|_{p, \Omega} \\
& \leq\|f\|_{p_{1}, \Omega}\|g\|_{p_{2}, \Omega}+\left(\|g\|_{p_{2}, \Omega}\|\nabla f\|_{p_{1}, \Omega}+\|f\|_{p_{1}, \Omega}\|\nabla g\|_{p_{2}, \Omega}\right) \\
& \leq\|f\|_{1, p_{1}, \Omega}\|g\|_{1, p_{2}, \Omega},
\end{aligned}
$$

which proves $(2.2)$.

We now prove Theorem 1.1.

Proof of Theorem 1.1. It follows from (1.1) that

$$
\left|\nabla M_{\Omega} f(x)\right| \leq 2 M_{\Omega}|\nabla f|(x),
$$

for almost every $x \in \Omega$. Invoking Lemma 2.2 and (2.4), one has

$$
\begin{aligned}
& \left|\nabla\left[b, M_{\Omega}\right](f)(x)\right|=\left|\nabla\left(b M_{\Omega} f\right)(x)-\nabla M_{\Omega}(b f)(x)\right| \\
& \leq|\nabla b(x)|\left|M_{\Omega} f(x)\right|+|b(x)|\left|\nabla M_{\Omega} f(x)\right|+2 M_{\Omega}|\nabla(b f)|(x) \\
& \leq|\nabla b(x)|\left|M_{\Omega} f(x)\right|+2|b(x)| M_{\Omega}|\nabla f|(x)+2 M_{\Omega}|\nabla b f|(x)+2 M_{\Omega}|b \nabla f|(x),
\end{aligned}
$$

for almost every $x \in \Omega$, which proves (1.5). By (1.5), (1.2), Hölder's inequality and the $L^{p}$ bounds for $M_{\Omega}$, one can get

$$
\left\|\left[b, M_{\Omega}\right](f)\right\|_{1, p, \Omega}=\left\|\left[b, M_{\Omega}\right](f)\right\|_{p, \Omega}+\left\|\nabla\left[b, M_{\Omega}\right](f)\right\|_{p, \Omega} \leq C_{p_{1}, p_{2}, n}\|b\|_{1, p_{2}, \Omega}\|f\|_{1, p_{1}, \Omega},
$$

which proves (1.6). that

We now prove the continuity part. Let $f_{j} \rightarrow f$ in $W^{1, p_{1}}(\Omega)$. We want to show

$$
\left\|\left[b, M_{\Omega}\right]\left(f_{j}\right)-\left[b, M_{\Omega}\right](f)\right\|_{1, p, \Omega} \rightarrow 0 \text { as } j \rightarrow \infty .
$$

Invoking Lemma 2.2, we can get

$$
\left\|b f_{j}-b f\right\|_{1, p, \Omega}=\left\|b\left(f_{j}-f\right)\right\|_{1, p, \Omega} \leq\|b\|_{1, p_{2}, \Omega}\left\|f_{j}-f\right\|_{1, p_{1}, \Omega} .
$$

It was shown in [26, Theorem 2.12] that

$$
\left\|M_{\Omega} f_{j}-M_{\Omega} f\right\|_{1, p_{1}, \Omega} \rightarrow 0 \quad \text { as } j \rightarrow \infty .
$$

Combining (2.8) with (2.6) and (2.7) implies that

$$
\begin{gathered}
\left\|b M_{\Omega} f_{j}-b M_{\Omega} f\right\|_{1, p, \Omega} \rightarrow 0 \text { as } j \rightarrow \infty, \\
\left\|M_{\Omega}\left(b f_{j}\right)-M_{\Omega}(b f)\right\|_{1, p, \Omega} \rightarrow 0 \text { as } j \rightarrow \infty .
\end{gathered}
$$

Then (2.5) follows directly from (2.9) and (2.10). This completes the proof of Theorem 1.1.

\section{Proof of Theorem $\mathbf{1 . 2}$}

3.1. Preliminaries, notations and lemmas. Let us give some notations and lemmas. Set $\delta(x)=\operatorname{dist}\left(x, \Omega^{c}\right)$. According to Rademacher's theorem, as a Lipschitz function $\delta$ is differentiable almost everywhere in $\Omega$. Moreover, $|\nabla \delta(x)|=1$ for almost every $x \in \Omega$. Let $b, f$ be two suitable functions defined on $\Omega$. For $0<t<1$, we define the function $A_{t, b, f}: \Omega \rightarrow[-\infty, \infty]$ by

$$
A_{t, b, f}(x)=\frac{1}{|B(x, t \delta(x))|} \int_{B(x, t \delta(x))}|b(x)-b(y)| f(y) d y .
$$


We now establish the following result, which plays a pivotal role in the proof of Theorem 1.2.

Lemma 3.1. Let $f \in W^{1, p_{1}}(\Omega)$ and $b \in W^{1, p_{2}}(\Omega)$ with $1<p_{1}, p_{2}, p_{1} p_{2} /\left(p_{1}+p_{2}\right)<$ $\infty$. Assume that $|\Omega|<\infty$. Then $A_{t, b, f} \in W^{1, p}(\Omega)$ with $p=p_{1} p_{2} /\left(p_{1}+p_{2}\right)$ and

$$
\left|\nabla A_{t, b, f}(x)\right| \leq 2\left(M_{b, \Omega}|\nabla f|(x)+M_{\Omega}(|\nabla b| f)(x)\right)+|\nabla b|(x) M_{\Omega}|f|(x),
$$

for almost every $x \in \Omega$.

Proof. We divide the proof into two steps.

Step 1. The case $b \in W^{1, p_{2}}(\Omega) \cap \mathcal{C}^{\infty}(\Omega)$ and $f \in W^{1, p_{1}}(\Omega) \cap \mathcal{C}^{\infty}(\Omega)$. Let $\varpi_{n}=$ $|B(0,1)|$. Fix $i=1,2, \ldots, n$. By Leibniz rule, one gets

$$
\begin{aligned}
D_{i} A_{t, b, f}(x)= & D_{i}\left(\frac{1}{\varpi_{n}(t \delta(x))^{n}}\right) \int_{B(x, t \delta(x))}|b(x)-b(y)| f(y) d y \\
& +\frac{1}{\varpi_{n}(t \delta(x))^{n}} \cdot D_{i}\left(\int_{B(x, t \delta(x))}|b(x)-b(y)| f(y) d y\right)
\end{aligned}
$$

for almost every $x \in \Omega$. For convenience, we denote by $D_{i, x} F$ the $i$-th weak partial derivative of $F$ in $x$. By the chain rule and the fact that

$$
\frac{\partial}{\partial r} \int_{B(x, r)} f(y) d y=\int_{\partial B(x, r)} f(y) d \mathcal{H}^{n-1}(y),
$$

one obtains

$$
\begin{aligned}
& D_{i}\left(\int_{B(x, t \delta(x))}|b(x)-b(y)| f(y) d y\right) \\
& =\int_{B(x, t \delta(x))} D_{i, x}|b(x)-b(y)| f(y) d y+\int_{B(x, t \delta(x))} D_{i, y}(|b(x)-b(y)| f(y)) d y \\
& \quad+t \int_{\partial B(x, t \delta(x))}|b(x)-b(y)| f(y) d \mathcal{H}^{n-1}(y) \cdot D_{i} \delta(x)
\end{aligned}
$$

for almost every $x \in \Omega$, where $d \mathcal{H}^{n-1}$ is the normalized $(n-1)$-dimensional Hausdorff measure. Equalities (3.3) and (3.4) yield that

$$
\begin{aligned}
\nabla A_{t, b, f}(x)= & \frac{-n \nabla \delta(x)}{\delta(x)} \frac{1}{|B(x, t \delta(x))|} \int_{B(x, t \delta(x))}|b(x)-b(y)| f(y) d y \\
& +\frac{1}{|B(x, t \delta(x))|}\left(\int_{B(x, t \delta(x))} \nabla_{x}|b(x)-b(y)| f(y) d y\right. \\
& \left.+\int_{B(x, t \delta(x))} \nabla_{y}(|b(x)-b(y)| f(y)) d y\right) \\
& +t \int_{\partial B(x, t \delta(x))}|b(x)-b(y)| f(y) d \mathcal{H}^{n-1}(y) \cdot \nabla \delta(x) \\
= & \frac{n \nabla \delta(x)}{\delta(x)}\left(\frac{1}{|\partial B(x, t \delta(x))|} \int_{\partial B(x, t \delta(x))}|b(x)-b(y)| f(y) d \mathcal{H}^{n-1}(y)\right. \\
& \left.-\frac{1}{|B(x, t \delta(x))|} \int_{B(x, t \delta(x))}|b(x)-b(y)| f(y) d y\right)
\end{aligned}
$$




$$
\begin{aligned}
& +\frac{1}{|B(x, t \delta(x))|}\left(\int_{B(x, t \delta(x))} \nabla_{x}|b(x)-b(y)| f(y) d y\right. \\
& \left.+\int_{B(x, t \delta(x))} \nabla_{y}(|b(x)-b(y)| f(y)) d y\right)
\end{aligned}
$$

for almost every $x \in \Omega$. Here $\nabla_{x}=\left(D_{1, x}, \ldots, D_{n, x}\right)$ and $\nabla_{y}=\left(D_{1, y}, \ldots, D_{n, y}\right)$.

Fix $x \in \Omega$. Let $R>0$ be such that $B(x, R) \subset \Omega$ and $F(x, y)$ be a function defined on $\Omega \times \Omega$. By Green's first identity, one has

$$
\begin{aligned}
& \int_{\partial B(x, R)} F(x, y) \frac{\partial \mu}{\partial \nu}(y) d \mathcal{H}^{n-1}(y) \\
& =\int_{B(x, R)}\left(F(x, y) \triangle \mu(y)+\nabla_{y} F(x, y) \cdot \nabla \mu(y)\right) d y,
\end{aligned}
$$

where $\nu(y)=\frac{y-x}{R}$ is the unit outer normal of $B(x, R)$ and $\mu$ is a suitable function. Take $\mu(y)=\frac{|y-x|^{2}}{2}$. Then $\nabla \mu(y)=y-x, \triangle \mu(y)=n$ and $\frac{\partial \mu}{\partial \nu}(y)=R$. These facts together with (3.6) imply that

$$
\begin{aligned}
& \frac{1}{|\partial B(x, R)|} \int_{\partial B(x, R)} F(x, y) d \mathcal{H}^{n-1}(y)-\frac{1}{|B(x, R)|} \int_{B(x, R)} F(x, y) d y \\
& =\frac{1}{n} \frac{1}{|B(x, R)|} \int_{B(x, R)} \nabla_{y} F(x, y) \cdot(y-x) d y .
\end{aligned}
$$

Applying (3.7) with $R=t \delta(x)$ and $F(x, y)=|b(x)-b(y)| f(y)$, we get from (3.5) that

$$
\begin{aligned}
\left|\nabla A_{t, b, f}(x)\right| \leq & \frac{|\nabla \delta(x)|}{\delta(x)} \frac{1}{|B(x, t \delta(x))|} \int_{B(x, t \delta(x))}\left|\nabla_{y}(|b(x)-b(y)| f(y)) \cdot(y-x)\right| d y \\
& +\frac{1}{|B(x, t \delta(x))|}\left(\int_{B(x, t \delta(x))}\left|\nabla_{x}\right| b(x)-b(y)|f(y)| d y\right. \\
& \left.+\int_{B(x, t \delta(x))}\left|\nabla_{y}(|b(x)-b(y)| f(y))\right| d y\right) \\
\leq & \frac{2}{|B(x, t \delta(x))|} \int_{B(x, t \delta(x))}\left|\nabla_{y}(|b(x)-b(y)| f(y))\right| d y \\
& +\frac{1}{|B(x, t \delta(x))|} \int_{B(x, t \delta(x))}\left|\nabla_{x}\right| b(x)-b(y)|f(y)| d y .
\end{aligned}
$$

Since $|\Omega|<\infty$, we have that $b(x)-b(\cdot) \in W^{1, p_{2}}(\Omega)$ and $|b(x)-b(\cdot)| \in W^{1, p_{2}}(\Omega)$. Invoking Lemma 2.2, we have that $|b(x)-b(\cdot)| f(\cdot) \in W^{1, p}(\Omega)$ and $\nabla_{y}(\mid b(x)-$ $b(y) \mid f(y))=f(y) \nabla_{y}(|b(x)-b(y)|)+|b(x)-b(y)| \nabla_{y} f(y)$ for almost every $y \in \Omega$. Moreover, $\left|\nabla_{y}\right| b(x)-b(y)||=|\nabla b(y)|$ for almost every $y \in \Omega$ and $\left|\nabla_{x}\right| b(x)-b(y)||=|\nabla b(x)|$ for almost every $x \in \Omega$. These facts together with (3.8) lead to

$$
\begin{aligned}
\left|\nabla A_{t, b, f}(x)\right| \leq & \frac{2}{|B(x, t \delta(x))|} \int_{B(x, t \delta(x))}(|b(x)-b(y)||\nabla f(y)|+|\nabla b(y)||f(y)|) d y \\
& +|\nabla b(x)| \frac{1}{|B(x, t \delta(x))|} \int_{B(x, t \delta(x))}|f(y)| d y \\
\leq & 2 M_{b, \Omega}|\nabla f|(x)+2 M_{\Omega}(|\nabla b| f)(x)+|\nabla b|(x) M_{\Omega} f(x),
\end{aligned}
$$


for almost every $x \in \Omega$. This proves (3.2) for the case $b \in W^{1, p_{2}}(\Omega) \cap \mathcal{C}^{\infty}(\Omega)$ and $f \in W^{1, p_{1}}(\Omega) \cap \mathcal{C}^{\infty}(\Omega)$.

Step 2. The general case. The proof in the general case follows from an approximation argument. To this end, we first assume that $b \in W^{1, p_{2}}(\Omega)$ and $f \in W^{1, p_{1}}(\Omega)$ for some $p_{1}, p_{2}$ with $1<p_{1}, p_{2}, p<\infty$ and $1 / p=1 / p_{1}+1 / p_{2}$. There exist two sequences of functions $\left\{\varphi_{j}\right\}_{j=1}^{\infty}$ in $W^{1, p_{1}}(\Omega) \cap \mathcal{C}^{\infty}(\Omega)$ and $\left\{\psi_{j}\right\}_{j=1}^{\infty}$ in $W^{1, p_{2}}(\Omega) \cap \mathcal{C}^{\infty}(\Omega)$ such that $\varphi_{j} \rightarrow f$ in $W^{1, p_{1}}(\Omega)$ and $\psi_{j} \rightarrow b$ in $W^{1, p_{2}}(\Omega)$ as $j \rightarrow \infty$. Then there exists a subsequence $\left\{\psi_{j_{k}}\right\}_{k=1}^{\infty} \subset\left\{\psi_{j}\right\}_{j=1}^{\infty}$ such that $\psi_{j_{k}}(x) \rightarrow b(x)$ as $k \rightarrow \infty$ for almost every $x \in \Omega$. Fix $t \in(0,1)$. Let

$$
v_{k, t}(x)=\frac{1}{|B(x, t \delta(x))|} \int_{B(x, t \delta(x))}\left|\psi_{j_{k}}(x)-\psi_{j_{k}}(y)\right| \varphi_{j_{k}}(y) d y .
$$

Note that

$$
\begin{aligned}
\left|v_{k, t}(x)-A_{t, b, f}(x)\right| \leq & \frac{\left|\psi_{j_{k}}(x)-b(x)\right|+|b(x)|}{|B(x, t \delta(x))|} \int_{B(x, t \delta(x))}\left|\varphi_{j_{k}}(y)-f(y)\right| d y \\
& +\frac{\left|\psi_{j_{k}}(x)-b(x)\right|}{|B(x, t \delta(x))|} \int_{B(x, t \delta(x))}|f(y)| d y \\
& +\frac{1}{|B(x, t \delta(x))|} \int_{B(x, t \delta(x))}\left|\psi_{j_{k}}(y)-b(y)\right|\left|\varphi_{j_{k}}(y)-f(y)\right| d y \\
& +\frac{1}{|B(x, t \delta(x))|} \int_{B(x, t \delta(x))}\left|\psi_{j_{k}}(y)-b(y) \| f(y)\right| d y \\
& +\frac{1}{|B(x, t \delta(x))|} \int_{B(x, t \delta(x))}\left|b(y) \| \varphi_{j_{k}}(y)-f(y)\right| d y \\
\leq & |B(x, t \delta(x))|^{-1 / p_{1}} \mid\left(\left(\left|\psi_{j_{k}}(x)-b(x)\right|+|b(x)|\right)\left\|\varphi_{j_{k}}-f\right\|_{p_{1}, \Omega}\right. \\
& \left.+\left|\psi_{j_{k}}(x)-b(x)\right|\|f\|_{p_{1}, \Omega}\right) \\
& +|B(x, t \delta(x))|^{-1 / p}\left(\left\|\psi_{j_{k}}-b\right\|_{p_{2}, \Omega}\left\|\varphi_{j_{k}}-f\right\|_{p_{1}, \Omega}\right. \\
& \left.+\left\|\psi_{j_{k}}-b\right\|_{p_{2}, \Omega}\|f\|_{p_{1}, \Omega}+\|b\|_{p_{2}, \Omega}\left\|\varphi_{j_{k}}-f\right\|_{p_{1}, \Omega}\right) .
\end{aligned}
$$

Therefore, for almost every $x \in \Omega$, it holds that

$$
\lim _{k \rightarrow \infty} v_{k, t}(x)=A_{t, b, f}(x)
$$

It is clear that $\left|v_{k, t}(x)\right| \leq M_{\psi_{j_{k}}, \Omega} \varphi_{j_{k}}(x)$ and

$$
\left|\nabla v_{k, t}(x)\right| \leq 2\left(M_{\psi_{j_{k}}, \Omega}\left|\nabla \varphi_{j_{k}}\right|(x)+M_{\Omega}\left(\left|\nabla \psi_{j_{k}}\right| \varphi_{j_{k}}\right)(x)\right)+\left|\nabla \psi_{j_{k}}\right|(x) M_{\Omega}\left|\varphi_{j_{k}}\right|(x),
$$

for almost every $x \in \Omega$. By (3.9), (1.3), Hölder's inequality and the boundedness for $M_{\Omega}$, we have

$$
\begin{aligned}
\left\|v_{k, t}\right\|_{1, p, \Omega}= & \left\|v_{k, t}\right\|_{p, \Omega}+\left\|\nabla v_{k, t}\right\|_{p, \Omega} \\
\leq & \left\|M_{\psi_{j_{k}}, \Omega} \varphi_{j_{k}}\right\|_{p, \Omega}+2\left\|M_{\psi_{j_{k}}, \Omega}\left|\nabla \varphi_{j_{k}}\right|\right\|_{p, \Omega}+2\left\|M_{\Omega}\left(\left|\nabla \psi_{j_{k}}\right| \varphi_{j_{k}}\right)\right\|_{p, \Omega} \\
& +\left\|\left|\nabla \psi_{j_{k}}\right| M_{\Omega}\left|\varphi_{j_{k}}\right|\right\|_{p, \Omega} \\
\leq & C_{p_{1}, p_{2}, n}\left\|\psi_{j_{k}}\right\|_{p_{2}, \Omega}\left\|\varphi_{j_{k}}\right\|_{p_{1}, \Omega}+C_{p_{1}, p_{2}, n}\left\|\psi_{j_{k}}\right\|_{p_{2}, \Omega}\left\|\nabla \varphi_{j_{k}}\right\|_{p_{1}, \Omega} \\
& +C_{p, n}\left\|\left|\nabla \psi_{j_{k}}\right| \varphi_{j_{k}}\right\|_{p, \Omega}+\left\|\nabla \psi_{j_{k}}\right\|_{p_{2}, \Omega}\left\|M_{\Omega}\left|\varphi_{j_{k}}\right|\right\|_{p_{1}, \Omega} \\
\leq & C_{p_{1}, p_{2}, n}\left\|\psi_{j_{k}}\right\|_{1, p_{2}, \Omega}\left\|\varphi_{j_{k}}\right\|_{1, p_{1}, \Omega} .
\end{aligned}
$$

Hence, $\left\{v_{k, t}\right\}_{k=1}^{\infty}$ is a bounded sequence in $W^{1, p}(\Omega)$ and has a weakly converging subsequence $\left\{v_{k_{\ell}, t}\right\}_{\ell=1}^{\infty}$ of $\left\{v_{k, t}\right\}_{k=1}^{\infty}$. Since $v_{k, t}(x) \rightarrow A_{t, b, f}(x)$ as $k \rightarrow \infty$ for almost 
every $x \in \Omega$, we can conclude that the weak gradient $\nabla A_{t, b, f}$ exists almost everywhere in $\Omega$ and that $\nabla v_{k_{\ell}, t} \rightarrow \nabla A_{t, b, f}$ weakly in $L^{p}(\Omega)$ as $k \rightarrow \infty$.

On the other hand, one can easily check that

$$
\begin{aligned}
& \left|M_{\psi_{j_{k}}, \Omega}\right| \nabla \varphi_{j_{k}}\left|(x)-M_{b, \Omega}\right| \nabla f|(x)| \\
& \leq \sup _{0<r<\operatorname{dist}\left(x, \Omega^{c}\right)} \frac{1}{|B(x, r)|} \int_{B(x, r)}|| \psi_{j_{k}}(x)-\psi_{j_{k}}(y)|| \nabla \varphi_{j_{k}}(y) \mid \\
& \quad-|b(x)-b(y)||\nabla f(y)| \mid d y \\
& \leq\left|\psi_{j_{k}}(x)-b(x)\right| M_{\Omega}|\nabla f|(x)+M_{\Omega}\left(\left(\psi_{j_{k}}-b\right)|\nabla f|\right)(x) \\
& \quad+M_{\psi_{j_{k}}, \Omega}\left|\nabla\left(\varphi_{j_{k}}-f\right)\right|(x) .
\end{aligned}
$$

By (3.10), the sublinearity of $M_{b, \Omega}$, the $L^{p_{1}}$ bounds for $M_{\Omega}$, Hölder's inequality and (1.4), we can get

$$
\begin{aligned}
& \left\|M_{\psi_{j_{k}}, \Omega}\left|\nabla \varphi_{j_{k}}\right|-M_{b, \Omega}|\nabla f|\right\|_{p, \Omega} \\
\leq & \left\|\left(\psi_{j_{k}}-b\right)\left|M_{\Omega}\right| \nabla f \mid\right\|_{p, \Omega}+\left\|M_{\Omega}\left(\left(\psi_{j_{k}}-b\right)|\nabla f|\right)\right\|_{p, \Omega} \\
& +\left\|M_{\psi_{j_{k}}, \Omega}\left|\nabla\left(\varphi_{j_{k}}-f\right)\right|\right\|_{p, \Omega} \\
\leq & \left\|\psi_{j_{k}}-b\right\|_{p_{2}, \Omega}\left\|M_{\Omega}|\nabla f|\right\|_{p_{1}, \Omega}+C_{p_{n} n}\left\|\left(\psi_{j_{k}}-b\right)|\nabla f|\right\|_{p, \Omega} \\
& +C_{p_{1}, p_{2}, n}\left\|\psi_{j_{k}}\right\|_{p_{2}, \Omega}\left\|\nabla\left(\varphi_{j_{k}}-f\right)\right\|_{p_{1}, \Omega} \\
\leq & C_{p_{1}, p_{2}, n}\left\|\psi_{j_{k}}-b\right\|_{p_{2}, \Omega}\|\nabla f\|_{p_{1}, \Omega}+C_{p_{1}, p_{2}, n}\left\|\psi_{j_{k}}-b\right\|_{p_{2}, \Omega}\|\nabla f\|_{p_{1}, \Omega} \\
& +C_{p_{1}, p_{2}, n}\left\|\psi_{j_{k}}\right\|_{p_{2}, \Omega}\left\|\nabla\left(\varphi_{j_{k}}-f\right)\right\|_{p_{1}, \Omega} .
\end{aligned}
$$

The sublinearity and the bounds of $M_{\Omega}$ together with Hölder's inequality yield that

$$
\begin{aligned}
& \left\|M_{\Omega}\left(\left|\nabla \psi_{j_{k}}\right| \varphi_{j_{k}}\right)-M_{\Omega}(|\nabla b| f)\right\|_{p, \Omega} \\
& \leq\left\|M_{\Omega}\left(\left|\nabla \psi_{j_{k}}\right| \varphi_{j_{k}}-|\nabla b| f\right)\right\|_{p, \Omega} \\
& \leq C_{p, n}\left\|\left|\nabla \psi_{j_{k}}\right| \varphi_{j_{k}}-|\nabla b| f\right\|_{p, \Omega} \\
& \leq C_{p_{1}, p_{2}, n}\left\|\nabla\left(\psi_{j_{k}}-b\right)\right\|_{p_{2}, \Omega}\left\|\varphi_{j_{k}}\right\|_{p_{1}, \Omega}+\|\nabla b\|_{p_{2}, \Omega}\left\|\varphi_{j_{k}}-f\right\|_{p_{1}, \Omega}
\end{aligned}
$$

and

$$
\begin{aligned}
& \left\|\left|\nabla \psi_{j_{k}}\right| M_{\Omega} \varphi_{j_{k}}-|\nabla b| M_{\Omega} f\right\|_{p, \Omega} \\
& \leq C_{p_{1}, n}\left(\left\|\nabla \psi_{j_{k}}\right\|_{p_{2}, \Omega}\left\|\varphi_{j_{k}}-f\right\|_{p_{1}, \Omega}+\left\|\nabla\left(\psi_{j_{k}}-b\right)\right\|_{p_{2}, \Omega}\|f\|_{p_{1}, \Omega}\right) .
\end{aligned}
$$

Let $g_{\ell}=2\left(M_{\psi_{j_{l}}, \Omega}\left|\nabla \varphi_{j_{k_{\ell}}}\right|+M_{\Omega}\left(\left|\nabla \psi_{j_{k_{\ell}}}\right| \varphi_{j_{k_{\ell}}}\right)\right)+\left|\nabla \psi_{j_{k_{\ell}}}\right| M_{\Omega} \varphi_{j_{k_{\ell}}}$. It follows from (3.11)(3.13) that

$$
g_{\ell} \rightarrow 2\left(M_{b, \Omega}|\nabla f|+M_{\Omega}(|\nabla b| f)\right)+|\nabla b| M_{\Omega} f \text { in } L^{p}(\Omega) \text { as } \ell \rightarrow \infty .
$$

Applying (3.9) and Proposition 2.1 to (3.14) with $f_{\ell}=\left|\nabla v_{k_{\ell}, t}\right|$, we can get (3.2). This completes the proof of Lemma 3.1.

In order to prove the continuity result of Theorem 1.2, we need to introduce some notations and establish some lemmas.

For $A \subset \mathbb{R}^{n}$ and $x \in \mathbb{R}^{n}$, let $d(x, A):=\inf _{a \in A}|x-a|$ and $A_{(\lambda)}:=\{x \in$ $\left.\mathbb{R}^{n} ; d(x, A) \leq \lambda\right\}$ for $\lambda \geq 0$. The notation $K \subset \subset \Omega$ means that $K$ is open, bounded and $\bar{K} \subset \Omega$. Let $b \in L^{p_{2}}(\Omega)$ and $f \in L^{p_{1}}(\Omega)$ for some $1<p_{1}, p_{2}, p<\infty$ with $1 / p=$ $1 / p_{1}+1 / p_{2}$. For every $x \in \Omega$, we define the function $u_{x, b, f}(r):[0, \delta(x)] \rightarrow[-\infty, \infty]$ 
by

$$
\begin{aligned}
& u_{x, b, f}(0)=0, \\
& u_{x, b, f}(r)=\frac{1}{|B(x, r)|} \int_{B(x, r)}|b(x)-b(y)| f(y) d y, \quad r \in(0, \delta(x)] .
\end{aligned}
$$

Define the set $\mathcal{R}(f)(x)$ by

$$
\mathcal{R}(f)(x)=\left\{r \in[0, \delta(x)] ; M_{b, \Omega} f(x)=u_{x, b,|f|}(r)\right\} .
$$

For any $x \in \Omega$, we have that $|b(x)-b(\cdot)| \in L^{p_{2}}(\Omega)$ since $|\Omega|<\infty$. Thus $\mid b(x)-$ $b(\cdot) \mid f(\cdot) \in L^{p}(\Omega)$ by Hölder's inequality. By the Lebesgue differentiation theorem, we see that $\lim _{r \rightarrow 0^{+}} u_{x, b, f}(r)=0$ for almost everywhere $x \in \Omega$. It follows that the functions $u_{x, b, f}$ are continuous on $(0, \delta(x)]$ for all $x \in \Omega$ and at $r=0$ for almost every $x \in \Omega$.

Lemma 3.2. Let $1<p_{1}, p_{2}, p<\infty$ and $1 / p=1 / p_{1}+1 / p_{2}$. Let $b \in L^{p_{2}}(\Omega)$. Suppose that $f_{j} \rightarrow f$ in $L^{p_{1}}(\Omega)$ when $j \rightarrow \infty$. Let $\Omega_{R}=\Omega \cap B(0, R)$. Then for all $R>0$ and $\lambda>0$, it holds that

$$
\lim _{j \rightarrow \infty}\left|\left\{x \in \Omega_{R} ; \mathcal{R}\left(f_{j}\right)(x) \nsubseteq \mathcal{R}(f)(x)_{(\lambda)}\right\}\right|=0 .
$$

Proof. Let $R>0, \lambda>0$ and fix $\epsilon \in(0,1)$. Without loss of generality we may assume that all $f_{j}, f \geq 0$ since $\mathcal{R}(f)(x)=\mathcal{R}(|f|)(x)$ and $\left|f_{j}\right| \rightarrow|f|$ in $L^{p_{1}}(\Omega)$ as $j \rightarrow \infty$. By the arguments similar to those used in the proof of [25, Lemma 2.2], we see that the set $\left\{x \in \Omega_{R} ; \mathcal{R}\left(f_{j}\right)(x) \nsubseteq \mathcal{R}(f)(x)_{(\lambda)}\right\}$ is measurable for any $j \in \mathbb{Z}$ when all $f_{j}$ and $f$ are locally integrable functions. Moreover, for almost every $x \in \Omega_{R}$, there exists $\gamma(x) \in \mathbb{N} \backslash\{0\}$ such that

$$
u_{x, b, f}(r)<M_{b, \Omega} f(x)-(\gamma(x))^{-1}, \text { when } d(r, \mathcal{R}(f)(x))>\lambda .
$$

Otherwise, for almost every $x \in \Omega_{R}$, there exists a bounded sequence of radii $\left\{r_{k}\right\}_{k=1}^{\infty}$ such that

$$
\lim _{k \rightarrow \infty} u_{x, b, f}\left(r_{k}\right)=M_{b, \Omega} f(x) \text { and } d\left(r_{k}, \mathcal{R}(f)(x)\right)>\lambda .
$$

There exists a subsequence $\left\{s_{k}\right\}_{k=1}^{\infty}$ of $\left\{r_{k}\right\}_{k=1}^{\infty}$ such that $s_{k} \rightarrow r$ as $k \rightarrow \infty$. It follows that $r \in \mathcal{R}(f)(x)$ and $d(r, \mathcal{R}(f)(x)) \geq \lambda$, which is a contradiction. Thus, (3.16) holds.

It follows from (3.16) that there exist $\gamma=\gamma(\lambda, R, \epsilon) \in \mathbb{N} \backslash\{0\}$ and a measurable set $E$ with $|E|<\epsilon$ such that

$$
\begin{aligned}
& \Omega_{R} \subset\left\{x \in \Omega_{R}: u_{x, b, f}(r)<M_{b, \Omega} f(x)-\gamma^{-1}, \text { if } d(r, \mathcal{R}(f)(x))>\lambda\right\} \cup E \\
& \quad=: B \cup E .
\end{aligned}
$$

Fix $x \in \Omega_{R}$ and $r$ such that $d(r, \mathcal{R}(f)(x))>\lambda$. It is clear that

$$
\begin{aligned}
M_{b, \Omega} f(x)-u_{x, b, f}(r) \leq & \left|M_{b, \Omega} f_{j}(x)-M_{b, \Omega} f(x)\right|+\left|M_{b, \Omega} f_{j}(x)-u_{x, b, f_{j}}(r)\right| \\
& +\left|u_{x, b, f_{j}}(r)-u_{x, b, f}(r)\right|,
\end{aligned}
$$

which leads to

$$
B \subset A_{1, j} \cup A_{2, j} \cup A_{3, j},
$$

where

$$
A_{1, j}:=\left\{x \in \Omega_{R}:\left|M_{b, \Omega} f_{j}(x)-M_{b, \Omega} f(x)\right| \geq(4 \gamma)^{-1}\right\}
$$




$$
\begin{aligned}
& A_{2, j}:=\left\{x \in \Omega_{R}:\left|u_{x, b, f_{j}}(r)-u_{x, b, f}(r)\right| \geq(2 \gamma)^{-1} \text { for some } r\right. \\
&\text { such that } d(r, \mathcal{R}(f)(x))>\lambda\}, \\
& A_{3, j}:=\left\{x \in \Omega_{R}: u_{x, b, f_{j}}(r)<M_{b, \Omega} f_{j}(x)-(4 \gamma)^{-1}, \text { if } d(r, \mathcal{R}(f)(x))>\lambda\right\} .
\end{aligned}
$$

Observe that

$$
A_{3, j} \subset\left\{x \in \Omega_{R}: \mathcal{R}\left(f_{j}\right)(x) \subset \mathcal{R}(f)(x)_{(\lambda)}\right\},
$$

which together with (3.18) yields that

$$
\left\{x \in \Omega_{R} ; \mathcal{R}\left(f_{j}\right)(x) \nsubseteq \mathcal{R}(f)(x)_{(\lambda)}\right\} \subset E \cup A_{1, j} \cup A_{2, j} .
$$

By the sublinearity of $M_{b, \Omega}$, we can get

$$
A_{1, j} \subset\left\{x \in \Omega_{R}: M_{b, \Omega}\left(f_{j}-f\right)(x) \geq(4 \gamma)^{-1}\right\} .
$$

Similarly we can obtain

$$
A_{2, j} \subset\left\{x \in \Omega_{R}: M_{b, \Omega}\left(f_{j}-f\right)(x) \geq(2 \gamma)^{-1}\right\} .
$$

Since $f_{j} \rightarrow f$ in $L^{p_{1}}(\Omega)$ as $j \rightarrow \infty$, there exists $N_{0}=N_{0}(\epsilon, \gamma) \in \mathbb{N}$ such that

$$
\left\|f_{j}-f\right\|_{p_{1}, \Omega}<\gamma^{-1} \epsilon, \quad\left\|f_{j}\right\|_{p_{1}, \Omega} \leq\|f\|_{p_{1}, \Omega}+1
$$

for any $j \geq N_{0}$. Hence, we get from (3.20)-(3.22) that

$$
\begin{aligned}
& \left|\left\{x \in \Omega_{R} ; \mathcal{R}\left(f_{j}\right)(x) \nsubseteq \mathcal{R}(f)(x)_{(\lambda)}\right\}\right| \\
& \leq 2\left|\left\{x \in \Omega_{R}: M_{b, \Omega}\left(f_{j}-f\right)(x) \geq(4 \gamma)^{-1}\right\}\right|+|E| \\
& \leq 2(4 \gamma)^{p}\left\|M_{b, \Omega}\left(f_{j}-f\right)\right\|_{p, \Omega}^{p}+\epsilon \\
& \leq C_{p_{1}, p_{2}, n} \gamma^{p}\|b\|_{p_{2}, \Omega}^{p}\left\|f_{j}-f\right\|_{p_{1}, \Omega}^{p} \epsilon \leq C_{p_{1}, p_{2}, n} \epsilon
\end{aligned}
$$

for all $j \geq N_{0}$. This yields (3.15) and finishes the proof of Lemma 3.2.

For $1 \leq l \leq n$, let $e_{l}=(0, \ldots, 0,1,0, \ldots, 0)$ be the canonical $l$-th base vector in $\mathbb{R}^{n}$. For $h \neq 0,1 \leq p<\infty, f \in L^{p}(\Omega)$ and $l \in\{1,2, \ldots, n\}$, we define the functions $f_{h, l}$ and $f_{\tau(h), l}$ by setting $f_{h, l}(x)=\frac{f_{\tau(h), l}(x)-f(x)}{h}$ and $f_{\tau(h), l}(x)=f\left(x+h e_{l}\right)$. It is well known that $f_{\tau(h), l} \rightarrow f$ in $L^{p}(K)$ for all $K \subset \subset \Omega$ when $h \rightarrow 0$, and if $f \in W^{1, p}(\Omega)$ with $p>1$ we have that $f_{h, l} \rightarrow D_{l} f$ in $L^{p}(K)$ when $h \rightarrow 0$ (see $[11,7.11]$ ).

Let $A, B$ be two subsets of $\mathbb{R}^{n}$. The Hausdorff distance of $A$ and $B$ is defined by

$$
\pi(A, B):=\inf \left\{\delta>0: A \subset B_{(\delta)} \text { and } B \subset A_{(\delta)}\right\} .
$$

The following lemma tells us how close the sets $\mathcal{R}(f)(x)$ and $\mathcal{R}(f)\left(x+h e_{l}\right)$ are when $h$ is small enough.

Lemma 3.3. Let $1<p_{1}, p_{2}, p<\infty$ and $1 / p=1 / p_{1}+1 / p_{2}$. Let $f \in L^{p_{1}}(\Omega)$ and $b \in L^{p_{2}}(\Omega)$. Then for $K \subset \subset \Omega, \lambda>0$ and $l=1,2, \ldots, n$, it holds that

$$
\left|\left\{x \in K ; \pi\left(\mathcal{R}(f)(x), \mathcal{R}(f)\left(x+h e_{l}\right)\right)>\lambda\right\}\right| \rightarrow 0 \text { when } h \rightarrow 0 .
$$

Proof. Fix $1 \leq l \leq n$. To prove (3.23), it is enough to prove that

$$
\begin{aligned}
& \lim _{h \rightarrow 0}\left|\left\{x \in K: \mathcal{R}(f)\left(x+h e_{l}\right) \nsubseteq \mathcal{R}(f)(x)_{(\lambda)}\right\}\right|=0, \\
& \lim _{h \rightarrow 0}\left|\left\{x \in K: \mathcal{R}(f)(x) \nsubseteq \mathbb{R}(f)\left(x+h e_{l}\right)_{(\lambda)}\right\}\right|=0 .
\end{aligned}
$$

We only prove (3.24) and (3.25) is analogous. The proof is motivated by the idea in the proof of [26, Lemma 2.3]. Fix $\epsilon \in(0,1)$ and $\lambda>0$. Applying the same argument 
as in getting (3.17), there exist a positive integer $\gamma=\gamma(\lambda, \epsilon)$ and a measurable set $E$ with $|E|<\epsilon$ such that

(3.26) $K \subset\left\{x \in K: u_{x, b, f}(r)<M_{b, \Omega} f(x)-\gamma^{-1}\right.$, if $\left.d(r, \mathcal{R}(f)(x))>\lambda\right\} \cup E=: G \cup E$.

Fix $h \in \mathbb{R}$, and let

$$
\begin{aligned}
B_{1, h}:= & \left\{x \in K:\left|M_{b, \Omega} f\left(x+h e_{l}\right)-M_{b, \Omega} f(x)\right|>(4 \gamma)^{-1}\right\}, \\
B_{2, h}:= & \left\{x \in K:\left|b_{\tau(h), l}(x)-b(x)\right| M_{\Omega} f_{\tau(h), l}(x)+M_{\Omega}\left(\left(b_{\tau(h), l}-b\right) f_{\tau(h), l}\right)(x)\right. \\
& \left.+M_{b, \Omega}\left(f_{\tau(h), l}-f\right)(x)>(2 \gamma)^{-1}\right\}, \\
B_{3, h}:= & \left\{x \in \Omega: \exists r \in\left[\delta(x)-2|h|, \delta\left(x+h e_{l}\right)\right]\right. \text { such that } \\
& \left.\left|u_{x+h e_{l}, b, f}(r)-u_{x+h e_{l}, b, f}\left(\delta\left(x+h e_{l}\right)-|h|\right)\right|>(8 \gamma)^{-1}\right\} .
\end{aligned}
$$

Firstly we prove that

(3.27) $\left\{x \in K: \mathcal{R}(f)\left(x+h e_{l}\right) \nsubseteq \mathcal{R}(f)(x)_{(2 \lambda)}\right\} \subset B_{1, h} \cup B_{2, h} \cup\left(B_{3, h}-h e_{l}\right) \cup E=: B_{h}$

when $h$ is small enough. Choose $h_{0} \in(0, \lambda)$ such that $K_{\left(2 h_{0}\right)} \subset \Omega$. It suffices to show that for $x \in G \backslash B_{h}$ with $|h|<\frac{1}{2} \min \left\{h_{0}, \delta(x)\right\}$, there exists $r \in \mathcal{R}(f)\left(x+h e_{l}\right)$ such that $d(r, \mathcal{R}(f)(x)) \leq 2 \lambda$. Otherwise, assume that $d(r, \mathcal{R}(f)(x))>2 \lambda$. We will consider the following two cases:

Case (i): $r<\delta(x)-|h|$. It follows from (3.26) that

$$
\begin{aligned}
M_{b, \Omega} f\left(x+h e_{l}\right) & =u_{x+h e_{l}, b, f}(r) \leq u_{x+h e_{l}, b, f}(r)-u_{x, b, f}(r)+u_{x, b, f}(r) \\
& \leq\left|u_{x+h e_{l}, b, f}(r)-u_{x, b, f}(r)\right|+M_{b, \Omega} f(x)-\gamma^{-1} .
\end{aligned}
$$

Note that

$$
\begin{aligned}
\left|u_{x+h e_{l}, b, f}(t)-u_{x, b, f}(t)\right| & \leq \frac{1}{|B(x, t)|}\left|\int_{B\left(x+h e_{l}, t\right)}\right| b\left(x+h e_{l}\right)-b(y) \mid f(y) d y \\
& -\int_{B(x, t)}|b(x)-b(y)| f(y) d y \mid \\
\leq & \frac{1}{|B(x, t)|} \int_{B(x, t)}|| b_{\tau(h), l}(x)-b_{\tau(h), l}(y)\left|f_{\tau(h), l}(y)-\right| b(x)-b(y)|f(y)| d y \\
\leq & \frac{\left|b_{\tau(h), l}(x)-b(x)\right|}{|B(x, t)|} \int_{B(x, t)}\left|f_{\tau(h), l}(y)\right| d y \\
& \left.+\frac{1}{|B(x, t)|} \int_{B(x, t)} \mid b_{\tau(h), l}(y)\right)-b(y)|| f_{\tau(h), l}(y) \mid d y \\
& +\frac{1}{|B(x, t)|} \int_{B(x, t)}|b(x)-b(y)|\left|f_{\tau(h), l}(y)-f(y)\right| d y \\
\leq & \left|b_{\tau(h), l}(x)-b(x)\right| M_{\Omega} f_{\tau(h), l}(x)+M_{\Omega}\left(\left(b_{\tau(h), l}-b\right) f_{\tau(h), l}\right)(x) \\
& +M_{b, \Omega}\left(f_{\tau(h), l}-f\right)(x)
\end{aligned}
$$

for any $t \in\left(0, \min \left\{\delta(x), \delta\left(x+h e_{l}\right)\right\}\right)$. Combining (3.28) with (3.29) yields that

$$
\begin{aligned}
M_{b, \Omega} f\left(x+h e_{l}\right) \leq & \left|b_{\tau(h), l}(x)-b(x)\right| M_{\Omega} f_{\tau(h), l}(x)+M_{\Omega}\left(\left(b_{\tau(h), l}-b\right) f_{\tau(h), l}\right)(x) \\
& +M_{b, \Omega}\left(f_{\tau(h), l}-f\right)(x)+M_{b, \Omega} f(x)-\gamma^{-1} \\
\leq & (2 \gamma)^{-1}+M_{b, \Omega} f(x)-\gamma^{-1} \leq M_{b, \Omega} f(x)-(2 \gamma)^{-1} .
\end{aligned}
$$


This yields that $\left|M_{b, \Omega} f(x)-M_{b, \Omega} f\left(x+h e_{l}\right)\right| \geq(2 \gamma)^{-1}$, which yields $x \in B_{1, h}$ and a contradiction.

Case (ii): $r \in\left[\delta(x)-|h|, \delta\left(x+h e_{l}\right)\right]$. It is clear that $d(\delta(x)-|h|, \mathcal{R}(f)(x))>\lambda$, $\delta\left(x+h e_{l}\right)-|h|<\delta(x)$ and $\delta\left(x+h e_{l}\right)-r \in[0,2|h|]$. Hence,

$$
\left|r-\left(\delta\left(x+h e_{l}\right)-|h|\right)\right|=|| h\left|-\left(\delta\left(x+h e_{l}\right)-r\right)\right| \leq|h|
$$

and

$d\left(\delta\left(x+h e_{l}\right)-|h|, \mathcal{R}(f)(x)\right) \geq d(r, \mathcal{R}(f)(x))-\left|r-\left(\delta\left(x+h e_{l}\right)-|h|\right)\right|>2 \lambda-|h|>\lambda$.

Inequality (3.29) together with (3.26) implies that

$$
\begin{aligned}
& M_{b, \Omega} f\left(x+h e_{l}\right)=u_{x+h e_{l}, b, f}(r) \\
& \leq\left|u_{x+h e_{l}, b, f}(r)-u_{x+h e_{l}, b, f}\left(\delta\left(x+h e_{l}\right)-|h|\right)\right| \\
& \quad+\left|u_{x+h e_{l}, b, f}\left(\delta\left(x+h e_{l}\right)-|h|\right)-u_{x, b, f}\left(\delta\left(x+h e_{l}\right)-|h|\right)\right|+u_{x, b, f}\left(\delta\left(x+h e_{l}\right)-|h|\right) \\
& \leq(8 \gamma)^{-1}+(2 \gamma)^{-1}+M_{b, \Omega} f(x)-\gamma^{-1} \leq M_{b, \Omega} f(x)-(4 \gamma)^{-1} .
\end{aligned}
$$

This yields that $\left|M_{b, \Omega} f(x)-M_{b, \Omega} f\left(x+h e_{l}\right)\right|>(4 \gamma)^{-1}$ and further $x \in B_{1, h}$, which is a contradiction and (3.27) is proved.

Secondly we show that

$$
\lim _{h \rightarrow 0}\left|B_{h}\right|=0 .
$$

It is clear that $\left|B_{3, h}-h e_{l}\right| \rightarrow 0$ when $h \rightarrow 0$. Note that $M_{b, \Omega} f \in L^{p}(\Omega)$. It follows that $\left(M_{b, \Omega} f\right)_{\tau(h), l} \rightarrow M_{b, \Omega} f$ in $L^{p}(K)$ when $h \rightarrow 0$. Hence, one has

$$
\left|B_{1, h}\right| \leq(4 \gamma)^{p}\left\|\left(M_{b, \Omega} f\right)_{\tau(h), l}-M_{b, \Omega} f\right\|_{p, \Omega}^{p} \rightarrow 0 \text { as } h \rightarrow 0 .
$$

By (1.4), the $L^{p}$ bounds for $M_{\Omega}$ and Hölder's inequality, it holds that

$$
\begin{aligned}
\left|B_{2, h}\right| \leq & (4 \gamma)^{p}\left\|\left|b_{\tau(h), l}-b\right| M_{\Omega} f_{\tau(h), l}+M_{\Omega}\left(\left(b_{\tau(h), l}-b\right) f_{\tau(h), l}\right)+M_{b, \Omega}\left(f_{\tau(h), l}-f\right)\right\|_{p, \Omega}^{p} \\
\leq & C_{p, \gamma}\left(\left\|\left|b_{\tau(h), l}-b\right| M_{\Omega} f_{\tau(h), l}\right\|_{p, \Omega}^{p}+\left\|M_{\Omega}\left(\left(b_{\tau(h), l}-b\right) f_{\tau(h), l}\right)\right\|_{p, \Omega}^{p}\right. \\
& \left.+\left\|M_{b, \Omega}\left(f_{\tau(h), l}-f\right)\right\|_{p, \Omega}^{p}\right) \\
\leq & C_{p_{1}, p_{2}, n, \gamma}\left(\left\|b_{\tau(h), l}-b\right\|_{p_{2}, \Omega}^{p}\left\|f_{\tau(h), l}\right\|_{p_{1}, \Omega}^{p}+\|b\|_{p_{2}, \Omega}^{p}\left\|f_{\tau(h), l}-f\right\|_{p_{1}, \Omega}^{p}\right),
\end{aligned}
$$

which together with (3.31) leads to $\left|B_{1, h} \cup B_{2, h}\right| \rightarrow 0$ when $h \rightarrow 0$. Then (3.30) holds. Combining (3.27) with (3.30) yields (3.24). This finishes the proof of Lemma 3.3.

The following key lemma will play a pivotal role in the proof of the continuity of Theorem 1.2.

Lemma 3.4. Let $f \in W^{1, p_{1}}(\Omega)$ and $b \in W^{1, p_{2}}(\Omega)$ with $1<p_{1}, p_{2}, p_{1} p_{2} /\left(p_{1}+p_{2}\right)<$ $\infty$. Assume that $|\Omega|<\infty$, then

(i) For any $l \in\{1,2, \ldots, n\}$, almost every $x \in \Omega$ and $r \in \mathcal{R}(f)(x)$ with $0<r<$ $\delta(x)$, it holds that

$$
\begin{aligned}
D_{l} M_{b, \Omega} f(x)= & \frac{1}{|B(x, r)|} \int_{B(x, r)}\left(D_{l, y}(|b(x)-b(y)|)+D_{l, x}(|b(x)-b(y)|)|f(y)| d y\right. \\
& +\frac{1}{|B(x, r)|} \int_{B(x, r)}|b(x)-b(y)| D_{l}|f|(y) d y .
\end{aligned}
$$

(ii) For any $l \in\{1,2, \ldots, n\}$, almost every $x \in \Omega$ and $0 \in \mathcal{R}(f)(x)$, it holds that

$$
D_{l} M_{b, \Omega} f(x)=0 .
$$


Proof. Without loss of generality we may assume that $f \geq 0$. Fix $l \in\{1,2, \ldots, n\}$. For convenience, we define the function $F_{b}: \Omega \times \Omega \rightarrow \mathbb{R}$ by $F_{b}(x, y)=|b(x)-b(y)|$. Note that $F_{b}(x, \cdot) \in W^{1, p_{2}}(\Omega)$ for all $x \in \Omega$ and $F_{b}(\cdot, y) \in W^{1, p_{2}}(\Omega)$ for all $y \in \Omega$ since $|\Omega|<\infty$. Since $F_{b}(\cdot, y) \in W^{1, p_{2}}(\Omega)$ for all $y \in \Omega$, then for any fixed $y \in \Omega$, we have that $\left|\nabla_{x} F_{b}(x, y)\right|=|\nabla b(x)|$ for almost every $x \in \Omega$. Therefore, $\left|\nabla_{x} F_{b}(x, \cdot)\right| \in L^{p_{2}}(\Omega)$ because of $|\Omega|<\infty$. It follows that $\left|D_{l, x} F_{b}(x, \cdot)\right| \in L^{p_{2}}(\Omega)$. Let $K \subset \subset \Omega$. By Lemma 3.3, we can choose a sequence $\left\{s_{k}\right\}_{k=1}^{\infty}, s_{k}>0$ and $s_{k} \rightarrow 0$ such that

$$
\lim _{k \rightarrow \infty} \pi\left(\mathcal{R}(f)(x), \mathcal{R}(f)\left(x+s_{k} e_{l}\right)\right)=0
$$

for almost every $x \in K$.

For convenience, we define the functions

$$
\left(F_{x, b}\right)_{s_{k}, l}(y)=\frac{1}{s_{k}}\left(F_{b}\left(x, y+s_{k} e_{l}\right)-F_{b}(x, y)\right), \quad\left(F_{y, b}\right)_{s_{k}, l}(x)=\frac{1}{s_{k}}\left(F_{b}\left(x+s_{k} e_{l}, y\right)-F_{b}(x, y)\right) \text {. }
$$

Then we have

$$
\begin{aligned}
& \max \left\{\left\|f_{\tau\left(s_{k}\right), l}-f\right\|_{p_{1}, K},\left\|b_{\tau\left(s_{k}\right), l}-b\right\|_{p_{2}, K}\right\} \rightarrow 0 \text { as } k \rightarrow \infty, \\
& \max \left\{\left\|f_{s_{k}, l}-D_{l} f\right\|_{p_{1}, K},\left\|b_{s_{k}, l}-D_{l} b\right\|_{p_{2}, K}\right\} \rightarrow 0 \text { as } k \rightarrow \infty, \\
&\left\|\left(F_{x, b}\right)_{s_{k}, l}-D_{l, y} F_{b}(x, \cdot)\right\|_{p_{2}, K} \rightarrow 0 \text { as } k \rightarrow \infty, \\
&\left\|\left(F_{y, b}\right)_{s_{k}, l}-D_{l, x} F_{b}(\cdot, y)\right\|_{p_{2}, K} \rightarrow 0 \text { as } k \rightarrow \infty, \\
&\left\|M_{\Omega}\left(f_{\tau\left(s_{k}\right), l}-f\right)\right\|_{p_{1}, K} \rightarrow 0 \text { as } k \rightarrow \infty, \\
&\left\|M_{\Omega}\left(\left(f_{\tau\left(s_{k}\right), l}-f\right)\left(b_{s_{k}, l}-D_{l} b\right)\right)\right\|_{p, K} \rightarrow 0 \text { as } k \rightarrow \infty, \\
&\left\|M_{\Omega}\left(f\left(b_{s_{k}, l}-D_{l} b\right)\right)\right\|_{p, K} \rightarrow 0 \text { as } k \rightarrow \infty, \\
&\left\|M_{\Omega}\left(\left(\left(F_{x, b}\right)_{s_{k}, l}-D_{l, y} F_{b}(x, \cdot)\right)\left(f_{\tau\left(s_{k}\right), l}-f\right)\right)\right\|_{p, K} \rightarrow 0 \text { as } k \rightarrow \infty, \\
&\left\|M_{\Omega}\left(\left(\left(F_{x, b}\right)_{s_{k}, l}-D_{l, y} F_{b}(x, \cdot)\right) f\right)\right\|_{p, K} \rightarrow 0 \text { as } k \rightarrow \infty, \\
&\left\|M_{\Omega}\left(D_{l, y} F_{b}(x, \cdot)\left(f_{\tau\left(s_{k}\right), l}-f\right)\right)\right\|_{p, K} \rightarrow 0 \text { as } k \rightarrow \infty, \\
&\left\|M_{D_{l} b, \Omega}\left(f_{\tau\left(s_{k}\right), l}-f\right)\right\|_{p, K} \rightarrow 0 \text { as } k \rightarrow \infty, \\
&\left\|M_{b, \Omega}\left(f_{s_{k}, l}-D_{l} f\right)\right\|_{p, K} \rightarrow 0 \text { as } k \rightarrow \infty .
\end{aligned}
$$

By the boundedness result in Theorem 1.2, we have that $M_{b, \Omega} f \in W^{1, p}(\Omega)$. Furthermore, we get

$$
\left\|\left(M_{b, \Omega} f\right)_{s_{k}, l}-D_{l} M_{b, \Omega} f\right\|_{p, K} \rightarrow 0 \text { as } k \rightarrow \infty .
$$

From the above facts, we can conclude that there exists a subsequence $\left\{h_{k}\right\}_{k=1}^{\infty}$ of $\left\{s_{k}\right\}_{k=1}^{\infty}$ and a measurable set $B_{1} \subset K$ such that $\left|K \backslash B_{1}\right|=0$ and for any $x \in B_{1}$, it holds that

$$
\begin{aligned}
& \lim _{k \rightarrow \infty} M_{\Omega}\left(\left(f_{\tau\left(h_{k}\right), l}-f\right)\left(b_{h_{k}, l}-D_{l} b\right)\right)(x)=0, \\
& \lim _{k \rightarrow \infty} M_{\Omega}\left(f\left(b_{h_{k}, l}-D_{l} b\right)\right)(x)=0, \\
& \lim _{k \rightarrow \infty} b_{h_{k}, l}(x)=D_{l} b(x), \\
& \lim _{k \rightarrow \infty} M_{\Omega}\left(f_{\tau\left(h_{k}\right), l}-f\right)(x)=0, \\
& \lim _{k \rightarrow \infty} M_{b, \Omega}\left(f_{h_{k}, l}-D_{l} f\right)(x)=0, \\
& \lim _{k \rightarrow \infty} M_{\Omega}\left(\left(\left(F_{x, b}\right)_{h_{k}, l}-D_{l, y} F_{b}(x, \cdot)\right)\left(f_{\tau\left(h_{k}\right), l}-f\right)\right)(x)=0, \\
& \lim _{k \rightarrow \infty} M_{\Omega}\left(\left(\left(F_{x, b}\right)_{h_{k}, l}-D_{l, y} F_{b}(x, \cdot)\right) f\right)(x)=0,
\end{aligned}
$$




$$
\begin{aligned}
& \lim _{k \rightarrow \infty}\left(F_{y, b}\right)_{h_{k}, l}(x)=D_{l, x} F_{b}(x, y) \text { for all } y \in \Omega, \\
& \lim _{k \rightarrow \infty} M_{D_{l} b, \Omega}\left(f_{\tau\left(h_{k}\right), l}-f\right)(x)=0, \\
& \lim _{k \rightarrow \infty} M_{\Omega}\left(D_{l, y} F_{b}(x, \cdot)\left(f_{\tau\left(h_{k}\right), l}-f\right)\right)(x)=0, \\
& \lim _{k \rightarrow \infty}\left(M_{b, \Omega}(f)\right)_{h_{k}, l}(x)=D_{l} M_{b, \Omega} f(x), \\
& \lim _{k \rightarrow \infty} \pi\left(\mathcal{R}(f)(x), \mathcal{R}(f)\left(x+h_{k} e_{l}\right)\right)=0 .
\end{aligned}
$$

We set

$$
\begin{aligned}
& B_{2}:=\left\{x \in K: M_{b, \Omega} f(x)=u_{x, b, f}(0) \text { if } 0 \in \mathcal{R}(f)(x)\right\}, \\
& B_{3}:=\bigcap_{k=1}^{\infty}\left\{x \in K: M_{b, \Omega} f\left(x+h_{k} e_{l}\right)=u_{x+h_{k} e_{l}, b, f}(0) \text { if } 0 \in \mathcal{R}(f)\left(x+h_{k} e_{l}\right)\right\}, \\
& B_{4}:=\left\{x \in K: \lim _{r \rightarrow 0} \frac{1}{|B(x, r)|} \int_{B(x, r)}|b(x)-b(y)| D_{l} f(y) d y=0\right\}, \\
& B_{5}:=\left\{x \in K: \lim _{r \rightarrow 0} \frac{1}{|B(x, r)|} \int_{B(x, r)}\left|D_{l} b(x)-D_{l} b(y)\right| f(y) d y=0\right\} .
\end{aligned}
$$

One can easily check that $\left|K \backslash B_{i}\right|=0$ for any $i=2,3,4,5$. Let $x \in \bigcap_{i=1}^{5} B_{i}$ and $r \in \mathcal{R}(f)(x)$ with $r<\delta(x)$. Since $\lim _{k \rightarrow \infty} \pi\left(\mathcal{R}(f)(x), \mathcal{R}(f)\left(x+h e_{k} e_{l}\right)\right)$, there exists radii $r_{k} \in \mathcal{R}(f)\left(x+h_{k} e_{l}\right)$ such that $\lim _{k \rightarrow \infty} r_{k}=r$. Without loss of generality we assume that all $r_{k}<\delta(x)$. We consider two cases:

Case A. $r>0$. In this case we may assume that all $r_{k} \in(0, \delta(x))$. We can write

$$
\begin{aligned}
& D_{l} M_{b, \Omega} f(x)=\lim _{k \rightarrow \infty} \frac{1}{h_{k}}\left(M_{b, \Omega} f\left(x+h_{k} e_{l}\right)-M_{b, \Omega} f(x)\right) \\
& \leq \lim _{k \rightarrow \infty} \frac{1}{h_{k}}\left(u_{x+h_{k} e_{l}, b, f}\left(r_{k}\right)-u_{x, b, f}\left(r_{k}\right)\right) \\
& =\lim _{k \rightarrow \infty} \frac{1}{\left|B\left(x, r_{k}\right)\right|} \int_{B\left(x, r_{k}\right)} \frac{F_{b}\left(x+h_{k} e_{l}, y+h_{k} e_{l}\right) f_{\tau\left(h_{k}\right), l}(y)-F_{b}(x, y) f(y)}{h_{k}} d y \\
& =\lim _{k \rightarrow \infty} \frac{1}{\left|B\left(x, r_{k}\right)\right|} \int_{B\left(x, r_{k}\right)}|b(x)-b(y)| f_{h_{k}, l}(y) d y \\
& \quad+\lim _{k \rightarrow \infty} \frac{1}{\left|B\left(x, r_{k}\right)\right|} \int_{B\left(x, r_{k}\right)} \frac{F_{b}\left(x+h_{k} e_{l}, y+h_{k} e_{l}\right)-F_{b}(x, y)}{h_{k}} f_{\tau\left(h_{k}\right), l}(y) d y .
\end{aligned}
$$

By the fact that $|\Omega|<\infty$ and Hölder's inequality it holds easily that $\mid b(x)-$ $b(\cdot) \mid D_{l} f(\cdot) \in L^{1}(\Omega)$, which together with the fact that $\lim _{k \rightarrow \infty} r_{k}=r$ implies that $\lim _{k \rightarrow \infty} \frac{1}{\left|B\left(x, r_{k}\right)\right|} \int_{B\left(x, r_{k}\right)}|b(x)-b(y)| D_{l} f(y) d y=\frac{1}{|B(x, r)|} \int_{B(x, r)}|b(x)-b(y)| D_{l} f(y) d y$.

It follows that

$$
\begin{gathered}
\left|\lim _{k \rightarrow \infty} \frac{1}{\left|B\left(x, r_{k}\right)\right|} \int_{B\left(x, r_{k}\right)}\right| b(x)-b(y) \mid f_{h_{k}, l}(y) d y \\
-\frac{1}{|B(x, r)|} \int_{B(x, r)}|b(x)-b(y)| D_{l} f(y) d y \mid
\end{gathered}
$$




$$
\begin{aligned}
& \leq \lim _{k \rightarrow \infty} \frac{1}{\left|B\left(x, r_{k}\right)\right|} \int_{B\left(x, r_{k}\right)}|b(x)-b(y)|\left|f_{h_{k}, l}(y)-D_{l} f(y)\right| d y \\
& \leq \lim _{k \rightarrow \infty} M_{b, \Omega}\left(f_{h_{k}, l}-D_{l} f\right)(x)=0 .
\end{aligned}
$$

On the other hand, it is easy to see that

$$
\begin{aligned}
& \lim _{k \rightarrow \infty} \frac{1}{\left|B\left(x, r_{k}\right)\right|} \int_{B\left(x, r_{k}\right)} \frac{F_{b}\left(x+h_{k} e_{l}, y+h_{k} e_{l}\right)-F_{b}(x, y)}{h_{k}} f_{\tau\left(h_{k}\right), l}(y) d y \\
& =\lim _{k \rightarrow \infty} \frac{1}{\left|B\left(x, r_{k}\right)\right|} \int_{B\left(x, r_{k}\right)}\left(F_{x, b}\right)_{h_{k}, l}(y) f_{\tau\left(h_{k}\right), l}(y) d y \\
& \quad+\lim _{k \rightarrow \infty} \frac{1}{\left|B\left(x, r_{k}\right)\right|} \int_{B\left(x, r_{k}\right)}\left(F_{y+h_{k} e_{l}, b}\right)_{h_{k}, l}(x) f_{\tau\left(h_{k}\right), l}(y) d y .
\end{aligned}
$$

One can easily check that

$$
\lim _{k \rightarrow \infty} \frac{1}{\left|B\left(x, r_{k}\right)\right|} \int_{B\left(x, r_{k}\right)} D_{l, y} F_{b}(x, y) f(y) d y=\frac{1}{|B(x, r)|} \int_{B(x, r)} D_{l, y} F_{b}(x, y) f(y) d y
$$

since $D_{l, y} F_{b}(x, \cdot) f(\cdot) \in L^{1}(\Omega)$. Therefore, one has

$$
\begin{aligned}
& \lim _{k \rightarrow \infty} \frac{1}{\left|B\left(x, r_{k}\right)\right|} \int_{B\left(x, r_{k}\right)}\left(F_{x, b}\right)_{h_{k}, l}(y) f_{\tau\left(h_{k}\right), l}(y) d y \\
- & \frac{1}{|B(x, r)|} \int_{B(x, r)} D_{l, y} F_{b}(x, y) f(y) d y \mid \\
\leq & \lim _{k \rightarrow \infty} \frac{1}{\left|B\left(x, r_{k}\right)\right|} \int_{B\left(x, r_{k}\right)}\left|\left(F_{x, b}\right)_{h_{k}, l}(y) f_{\tau\left(h_{k}\right), l}(y)-D_{l, y} F_{b}(x, y) f(y)\right| d y \\
\leq & \lim _{k \rightarrow \infty} \frac{1}{\left|B\left(x, r_{k}\right)\right|} \int_{B\left(x, r_{k}\right)}\left|\left(F_{x, b}\right)_{h_{k}, l}(y)-D_{l, y} F_{b}(x, y)\right|\left|f_{\tau\left(h_{k}\right), l}(y)-f(y)\right| d y \\
& +\lim _{k \rightarrow \infty} \frac{1}{\left|B\left(x, r_{k}\right)\right|} \int_{B\left(x, r_{k}\right)}\left|\left(F_{x, b}\right)_{h_{k}, l}(y)-D_{l, y} F_{b}(x, y)\right||f(y)| d y \\
& +\lim _{k \rightarrow \infty} \frac{1}{\left|B\left(x, r_{k}\right)\right|} \int_{B\left(x, r_{k}\right)}\left|D_{l, y} F_{b}(x, y)\right|\left|f_{\tau\left(h_{k}\right), l}(y)-f(y)\right| d y \\
\leq & \lim _{k \rightarrow \infty}\left(M_{\Omega}\left(\left(\left(F_{x, b}\right)_{h_{k}, l}-D_{l, y} F_{b}(x, \cdot)\right)\left(f_{\tau\left(h_{k}\right), l}-f\right)\right)(x)\right. \\
& \left.+M_{\Omega}\left(\left(\left(F_{x, b}\right)_{h_{k}, l}-D_{l, y} F_{b}(x, \cdot)\right) f\right)(x)+M_{\Omega}\left(D_{l, y} F_{b}(x, \cdot)\left(f_{\tau\left(h_{k}\right), l}-f\right)\right)(x)\right) \\
= & 0 .
\end{aligned}
$$

We now prove that

$$
\begin{aligned}
& \lim _{k \rightarrow \infty} \frac{1}{\left|B\left(x, r_{k}\right)\right|} \int_{B\left(x, r_{k}\right)}\left(F_{y+h_{k} e_{l}, b}\right)_{h_{k}, l}(x) f_{\tau\left(h_{k}\right), l}(y) d y \\
& =\frac{1}{|B(x, r)|} \int_{B(x, r)} D_{l, x} F_{b}(x, y) f(y) d y .
\end{aligned}
$$

Note that

$$
\left|\left(F_{y+h_{k} e_{l}, b}\right)_{h_{k}, l}(x)\right| \leq \frac{\| b\left(x+h_{k} e_{l}\right)-b\left(y+h_{k} e_{l}\right)|-| b(x)-b\left(y+h_{k} e_{l}\right)||}{h_{k}} \leq\left|b_{h_{k}, l}(x)\right| .
$$


Sobolev boundedness and continuity for commutators of the local Hardy-Littlewood maximal function 219

It follows that

$$
\begin{aligned}
& \left|\lim _{k \rightarrow \infty} \frac{1}{\left|B\left(x, r_{k}\right)\right|} \int_{B\left(x, r_{k}\right)}\left(F_{y+h_{k} e_{l}, b}\right)_{h_{k}, l}(x)\left(f_{\tau\left(h_{k}\right), l}(y)-f(y)\right) d y\right| \\
& \leq \lim _{k \rightarrow \infty}\left(\left|b_{h_{k}, l}(x)-D_{l} b(x)\right|+\left|D_{l} b(x)\right|\right) M_{\Omega}\left(f_{\tau\left(h_{k}\right), l}-f\right)(x)=0 .
\end{aligned}
$$

Hence, to prove (3.38), it suffices to show that

$$
\begin{aligned}
& \lim _{k \rightarrow \infty} \frac{1}{\left|B\left(x, r_{k}\right)\right|} \int_{B\left(x, r_{k}\right)}\left(F_{y+h_{k} e_{l}, b}\right)_{h_{k}, l}(x) f(y) d y \\
& =\frac{1}{|B(x, r)|} \int_{B(x, r)} D_{l, x} F_{b}(x, y) f(y) d y .
\end{aligned}
$$

Note that $D_{l, x} F_{b}(x, \cdot) f(\cdot) \in L^{1}(\Omega)$. This together with the fact that $\lim _{k \rightarrow \infty} \chi_{B\left(x, r_{k}\right)}=$ $\chi_{B(x, r)}$ yields that

$$
\frac{1}{|B(x, r)|} \int_{B(x, r)} D_{l, x} F_{b}(x, y) f(y) d y=\lim _{k \rightarrow \infty} \frac{1}{\left|B\left(x, r_{k}\right)\right|} \int_{B\left(x, r_{k}\right)} D_{l, x} F_{b}(x, y) f(y) d y .
$$

It follows that

$$
\begin{aligned}
& \mid \lim _{k \rightarrow \infty} \frac{1}{\left|B\left(x, r_{k}\right)\right|} \int_{B\left(x, r_{k}\right)}\left(F_{y+h_{k} e_{l}, b}\right)_{h_{k}, l}(x) f(y) d y \\
& -\frac{1}{|B(x, r)|} \int_{B(x, r)} D_{l, x} F_{b}(x, y) f(y) d y \mid \\
& \leq \lim _{k \rightarrow \infty} \frac{1}{\left|B\left(x, r_{k}\right)\right|} \int_{B\left(x, r_{k}\right)}\left|\left(F_{y+h_{k} e_{l}, b}\right)_{h_{k}, l}(x)-D_{l, x} F_{b}(x, y)\right| f(y) d y \\
& \leq \lim _{k \rightarrow \infty} \frac{1}{\left|B\left(x, r_{k}\right)\right|} \int_{B\left(x, r_{k}\right)}\left|\left(F_{y+h_{k} e_{l}, b}\right)_{h_{k}, l}(x)-D_{l, x} F_{b}\left(x, y+h_{k} e_{l}\right)\right| f(y) d y \\
& \quad+\lim _{k \rightarrow \infty} \frac{1}{\left|B\left(x, r_{k}\right)\right|} \int_{B\left(x, r_{k}\right)}\left|D_{l, x} F_{b}\left(x, y+h_{k} e_{l}\right)-D_{l, x} F_{b}(x, y)\right| f(y) d y .
\end{aligned}
$$

Note that $r_{k} \in(0, \delta(x))$ for $k \geq 1$. Take $r<r^{\prime}<\delta(x)$ satisfying $h_{k}+r_{k}<r^{\prime}$ for large $k$. Hence $B\left(x, r_{k}\right) \subset B\left(x, r^{\prime}\right) \subset \subset \Omega$ and $B\left(x+h_{k} e_{l}, r_{k}\right) \subset \Omega$. By Hölder's inequality and the change of variables, one has

$$
\begin{aligned}
& \frac{1}{\left|B\left(x, r_{k}\right)\right|} \int_{B\left(x, r_{k}\right)}\left|\left(F_{y+h_{k} e_{l}, b}\right)_{h_{k}, l}(x)-D_{l, x} F_{b}\left(x, y+h_{k} e_{l}\right)\right| f(y) d y \\
& \leq\left|B\left(x, r_{k}\right)\right|^{-1 / p}\left(\int_{B\left(x, r_{k}\right)}\left|\left(F_{y+h_{k} e_{l}, b}\right)_{h_{k}, l}(x)-D_{l, x} F_{b}\left(x, y+h_{k} e_{l}\right)\right|^{p_{2}} d y\right)^{1 / p_{2}} \\
& \quad \times\left(\int_{B\left(x, r_{k}\right)}|f(y)|^{p_{1}} d y\right)^{1 / p_{1}} \\
& \leq\left|B\left(x, r_{k}\right)\right|^{-1 / p}\left(\int_{B\left(x, r_{k}\right)}\left|\left(F_{y+h_{k} e_{l}, b}\right)_{h_{k}, l}(x)-D_{l, x} F_{b}\left(x, y+h_{k} e_{l}\right)\right|^{p_{2}} d y\right)^{1 / p_{2}} \\
& \quad \times\left(\int_{\Omega}|f(y)|^{p_{1}} d y\right)^{1 / p_{1}}
\end{aligned}
$$




$$
\begin{aligned}
& \leq\left|B\left(x, r_{k}\right)\right|^{-1 / p}\|f\|_{p_{1}, \Omega}\left(\int_{B\left(x+h_{k} e_{l}, r_{k}\right)}\left|\left(F_{y, b}\right)_{h_{k}, l}(x)-D_{l, x} F_{b}(x, y)\right|^{p_{2}} d y\right)^{1 / p_{2}} \\
& \leq\left|B\left(x, r_{k}\right)\right|^{-1 / p}\|f\|_{p_{1}, \Omega}\left\|\left(F_{\cdot, b}\right)_{h_{k}, l}(x)-D_{l, x} F_{b}(x, \cdot)\right\|_{p_{2}, B\left(x, r^{\prime}\right)} .
\end{aligned}
$$

Similarly we can get

$$
\begin{aligned}
& \frac{1}{\left|B\left(x, r_{k}\right)\right|} \int_{B\left(x, r_{k}\right)}\left|D_{l, x} F_{b}\left(x, y+h_{k} e_{l}\right)-D_{l, x} F_{b}(x, y)\right| f(y) d y \\
& \leq\left|B\left(x, r_{k}\right)\right|^{-1 / p}\|f\|_{p_{1}, \Omega}\left\|\left(D_{l, x} F_{b}\right)_{\tau\left(h_{k}\right), l}(x, \cdot)-D_{l, x} F_{b}(x, \cdot)\right\|_{p_{2}, B\left(x, r^{\prime}\right)} .
\end{aligned}
$$

Since $B\left(x, r^{\prime}\right) \subset \subset \Omega$, in the proof of Lemma 3.4 we noted that

$$
\lim _{k \rightarrow \infty}\left\|\left(F_{\cdot, b}\right)_{h_{k}, l}(x)-D_{l, x} F_{b}(x, \cdot)\right\|_{p_{2}, B\left(x, r^{\prime}\right)}=0,
$$

which together with (3.42) leads to

$$
\lim _{k \rightarrow \infty} \frac{1}{\left|B\left(x, r_{k}\right)\right|} \int_{B\left(x, r_{k}\right)}\left|\left(F_{y+h_{k} e_{l}, b}\right)_{h_{k}, l}(x)-D_{l, x} F_{b}\left(x, y+h_{k} e_{l}\right)\right| f(y) d y=0 .
$$

Since $\left|D_{l, x} F_{b}(x, \cdot)\right| \in L^{p_{2}}(\Omega)$, then

$$
\left\|\left(D_{l, x} F_{b}\right)_{\tau\left(h_{k}\right), l}(x, \cdot)-D_{l, x} F_{b}(x, \cdot)\right\|_{p_{2}, B\left(x, r^{\prime}\right)} \rightarrow 0 \text { as } k \rightarrow \infty .
$$

This together with (3.43) implies that

$$
\lim _{k \rightarrow \infty} \frac{1}{\left|B\left(x, r_{k}\right)\right|} \int_{B\left(x, r_{k}\right)}\left|D_{l, x} F_{b}\left(x, y+h_{k} e_{l}\right)-D_{l, x} F_{b}(x, y)\right| f(y) d y=0 .
$$

Combining (3.45) with (3.44) and (3.41) yields (3.40). It follows from (3.34)-(3.38) that

$$
\begin{aligned}
D_{l} M_{b, \Omega} f(x) \leq & \frac{1}{|B(x, r)|} \int_{B(x, r)}\left(D_{l, y}(|b(x)-b(y)|)+D_{l, x}(|b(x)-b(y)|)\right) f(y) d y \\
& +\frac{1}{|B(x, r)|} \int_{B(x, r)}|b(x)-b(y)| D_{l} f(y) d y
\end{aligned}
$$

On the other hand, we obtain that

$$
\begin{aligned}
& D_{l} M_{b, \Omega}(f)(x)=\lim _{k \rightarrow \infty} \frac{1}{h_{k}}\left(M_{b, \Omega} f\left(x+h_{k} e_{l}\right)-M_{b, \Omega} f(x)\right) \\
& \geq \lim _{k \rightarrow \infty} \frac{1}{h_{k}}\left(u_{x+h_{k} e_{l}, b, f}(r)-u_{x, b, f}(r)\right) \\
& =\lim _{k \rightarrow \infty} \frac{1}{|B(x, r)|} \int_{B(x, r)} \frac{F_{b}\left(x+h_{k} e_{l}, y+h_{k} e_{l}\right) f_{\tau\left(h_{k}\right), l}(y)-F_{b}(x, y) f(y)}{h_{k}} d y \\
& =\lim _{k \rightarrow \infty} \frac{1}{|B(x, r)|} \int_{B(x, r)}|b(x)-b(y)| f_{h_{k}, l}(y) d y \\
& \quad+\lim _{k \rightarrow \infty} \frac{1}{|B(x, r)|} \int_{B(x, r)} \frac{F_{b}\left(x+h_{k} e_{l}, y+h_{k} e_{l}\right)-F_{b}(x, y)}{h_{k}} f_{\tau\left(h_{k}\right), l}(y) d y .
\end{aligned}
$$


Sobolev boundedness and continuity for commutators of the local Hardy-Littlewood maximal function 221

Similar arguments to those in getting (3.35), (3.37) and (3.38) give that

$$
\begin{aligned}
& \lim _{k \rightarrow \infty} \frac{1}{|B(x, r)|} \int_{B(x, r)}|b(x)-b(y)| f_{h_{k}, l}(y) d y \\
& =\frac{1}{|B(x, r)|} \int_{B(x, r)}|b(x)-b(y)| D_{l} f(y) d y \\
& \lim _{k \rightarrow \infty} \frac{1}{|B(x, r)|} \int_{B(x, r)}\left(F_{x, b}\right)_{h_{k}, l}(y) f_{\tau\left(h_{k}\right), l}(y) d y \\
& =\frac{1}{|B(x, r)|} \int_{B(x, r)} D_{l, y} F_{b}(x, y) f(y) d y, \\
& \lim _{k \rightarrow \infty} \frac{1}{|B(x, r)|} \int_{B(x, r)}\left(F_{\left.y+h_{k} e_{l}, b\right)_{h_{k}, l}(x) f_{\tau\left(h_{k}\right), l}(y) d y}\right. \\
& =\frac{1}{|B(x, r)|} \int_{B(x, r)} D_{l, x} F_{b}(x, y) f(y) d y .
\end{aligned}
$$

Observe that

$$
\begin{aligned}
& \lim _{k \rightarrow \infty} \frac{1}{|B(x, r)|} \int_{B(x, r)} \frac{F_{b}\left(x+h_{k} e_{l}, y+h_{k} e_{l}\right)-F_{b}(x, y)}{h_{k}} f_{\tau\left(h_{k}\right), l}(y) d y \\
& =\lim _{k \rightarrow \infty} \frac{1}{|B(x, r)|} \int_{B(x, r)}\left(F_{x, b}\right)_{h_{k}, l}(y) f_{\tau\left(h_{k}\right), l}(y) d y \\
& \quad+\lim _{k \rightarrow \infty} \frac{1}{|B(x, r)|} \int_{B(x, r)}\left(F_{y+h_{k} e_{l}, b}\right)_{h_{k}, l}(x) f_{\tau\left(h_{k}\right), l}(y) d y .
\end{aligned}
$$

It follows from (3.47)-(3.51) that

$$
\begin{aligned}
D_{l} M_{b, \Omega} f(x) \geq & \frac{1}{|B(x, r)|} \int_{B(x, r)}\left(D_{l, y}|b(x)-b(y)|+D_{l, x}|b(x)-b(y)|\right) f(y) d y \\
& +\frac{1}{|B(x, r)|} \int_{B(x, r)}|b(x)-b(y)| D_{l} f(y) d y .
\end{aligned}
$$

Combining (3.46) with (3.52) implies that (3.32) holds for almost every $x \in K$.

Case B. $r=0$. Since $0 \in \mathcal{R}(f)(x)$, it holds that $M_{b, \Omega} f(x)=u_{x, b, f}(0)=0$. Then we have $|b(x)-b(y)| f(y)=0$ for almost every $y \in B(x, \delta(x))$. Hence, we can write

$$
D_{l} M_{b, \Omega} f(x)=\lim _{k \rightarrow \infty} \frac{1}{h_{k}} M_{b, \Omega} f\left(x+h_{k} e_{l}\right)=\lim _{k \rightarrow \infty} \frac{1}{h_{k}} u_{x+h_{k} e_{l}, b, f}\left(r_{k}\right) .
$$

If we have $r_{k}=0$ for infinitely many $k$, then we have $D_{l} M_{b, \Omega} f(x)=0$. Otherwise, there exists $k_{0} \in \mathbb{N}$ such that $r_{k}>0$ when $k \geq k_{0}$. Note that $|b(x)-b(y)| f(y)=0$ for almost every $y \in B(x, \delta(x))$. Then we have

$$
\begin{aligned}
& u_{x+h_{k}, b, f}\left(r_{k}\right)=\frac{1}{\left|B\left(x, r_{k}\right)\right|} \int_{B\left(x+h_{k} e_{l}, r_{k}\right)}\left|b\left(x+h_{k} e_{l}\right)-b(y)\right| f(y) d y \\
& =\frac{1}{\left|B\left(x, r_{k}\right)\right|} \int_{B\left(x, r_{k}\right)}\left|b\left(x+h_{k} e_{l}\right)-b\left(y+h_{k} e_{l}\right)\right| f\left(y+h_{k} e_{l}\right) d y \\
& \leq \frac{1}{\left|B\left(x, r_{k}\right)\right|} \int_{B\left(x, r_{k}\right)}\left|b\left(x+h_{k} e_{l}\right)-b\left(y+h_{k} e_{l}\right)-(b(x)-b(y))\right| f\left(y+h_{k} e_{l}\right) d y \\
& \quad+\frac{1}{\left|B\left(x, r_{k}\right)\right|} \int_{B\left(x, r_{k}\right)}|b(x)-b(y)|\left|f\left(y+h_{k} e_{l}\right)-f(y)\right| d y .
\end{aligned}
$$


It follows that

$$
\begin{aligned}
\frac{1}{h_{k}} u_{x+h_{k}, b, f}\left(r_{k}\right) \leq & \frac{1}{\left|B\left(x, r_{k}\right)\right|} \int_{B\left(x, r_{k}\right)}\left|b_{h_{k}, l}(x)-b_{h_{k}, l}(y)\right| f\left(y+h_{k} e_{l}\right) d y \\
& +\frac{1}{\left|B\left(x, r_{k}\right)\right|} \int_{B\left(x, r_{k}\right)}|b(x)-b(y)|\left|f_{h_{k}, l}(y)\right| d y .
\end{aligned}
$$

We can write

$$
\begin{aligned}
& \frac{1}{\left|B\left(x, r_{k}\right)\right|} \int_{B\left(x, r_{k}\right)}\left|b_{h_{k}, l}(x)-b_{h_{k}, l}(y)\right| f\left(y+h_{k} e_{l}\right) d y \\
\leq & \frac{1}{\left|B\left(x, r_{k}\right)\right|} \int_{B\left(x, r_{k}\right)}\left|b_{h_{k}, l}(x)-b_{h_{k}, l}(y)-\left(D_{l} b(x)-D_{l} b(y)\right)\right| f\left(y+h_{k} e_{l}\right) d y \\
& +\frac{1}{\left|B\left(x, r_{k}\right)\right|} \int_{B\left(x, r_{k}\right)}\left|D_{l} b(x)-D_{l} b(y)\right| f\left(y+h_{k} e_{l}\right) d y \\
\leq & \frac{\left|b_{h_{k}, l}(x)-D_{l} b(x)\right|}{\left|B\left(x, r_{k}\right)\right|} \int_{B\left(x, r_{k}\right)} f\left(y+h_{k} e_{l}\right) d y \\
& +\frac{1}{\left|B\left(x, r_{k}\right)\right|} \int_{B\left(x, r_{k}\right)}\left|b_{h_{k}, l}(y)-D_{l} b(y)\right| f\left(y+h_{k} e_{l}\right) d y \\
& +\frac{1}{\left|B\left(x, r_{k}\right)\right|} \int_{B\left(x, r_{k}\right)}\left|D_{l} b(x)-D_{l} b(y)\right|\left|f\left(y+h_{k} e_{l}\right)-f(y)\right| d y \\
& +\frac{1}{\left|B\left(x, r_{k}\right)\right|} \int_{B\left(x, r_{k}\right)}\left|D_{l} b(x)-D_{l} b(y)\right| f(y) d y \\
\leq & \left|b_{h_{k}, l}(x)-D_{l} b(x)\right|\left(M_{\Omega}\left(f_{\tau\left(h_{k}\right), l}-f\right)+M_{\Omega} f(x)\right) \\
& +\frac{1}{\left|B\left(x, r_{k}\right)\right|} \int_{B\left(x, r_{k}\right)}\left|b_{h_{k}, l}(y)-D_{l} b(y)\right|\left|f\left(y+h_{k} e_{l}\right)-f(y)\right| d y \\
& +\frac{1}{\left|B\left(x, r_{k}\right)\right|} \int_{B\left(x, r_{k}\right)}\left|b_{h_{k}, l}(y)-D_{l} b(y)\right| f(y) d y \\
& +M_{D_{l} b, \Omega}\left(f_{\tau\left(h_{k}\right), l}-f\right)(x)+\frac{1}{\left|B\left(x, r_{k}\right)\right|} \int_{B\left(x, r_{k}\right)}\left|D_{l} b(x)-D_{l} b(y)\right| f(y) d y \\
& +b_{h_{k}, l}(x)-D_{l} b(x) \mid\left(M_{\Omega}\left(f_{h_{k}, l}-D_{\tau} b\right)\left(f_{\tau\left(h_{k}\right), l}-f\left(f_{\tau\left(h_{k}\right), l}-f\right)(x)+M_{\Omega} f(x)\right)\right. \\
& +M_{\Omega}\left(\left(b_{h_{k}, l}-D_{l} b\right) f\right)(x) \\
& \int_{B\left(x, r_{k}\right)}\left|D_{l} b(x)-D_{l} b(y)\right| f(y) d y
\end{aligned}
$$

Consequently, one can get

$$
\lim _{k \rightarrow \infty} \frac{1}{\left|B\left(x, r_{k}\right)\right|} \int_{B\left(x, r_{k}\right)}\left|b_{h_{k}, l}(x)-b_{h_{k}, l}(y)\right| f\left(y+h_{k} e_{l}\right) d y=0
$$

On the other hand, we can get

$$
\frac{1}{\left|B\left(x, r_{k}\right)\right|} \int_{B\left(x, r_{k}\right)}|b(x)-b(y)|\left|f_{h_{k}, l}(y)\right| d y
$$




$$
\begin{aligned}
\leq & \frac{1}{\left|B\left(x, r_{k}\right)\right|} \int_{B\left(x, r_{k}\right)}|b(x)-b(y)|\left|f_{h_{k}, l}(y)-D_{l} f\right| d y \\
& +\frac{1}{\left|B\left(x, r_{k}\right)\right|} \int_{B\left(x, r_{k}\right)}|b(x)-b(y)|\left|D_{l} f(y)\right| d y \\
\leq & M_{b, \Omega}\left(f_{h_{k}, l}-D_{l} f\right)(x)+\frac{1}{\left|B\left(x, r_{k}\right)\right|} \int_{B\left(x, r_{k}\right)}|b(x)-b(y)|\left|D_{l} f(y)\right| d y .
\end{aligned}
$$

This yields

$$
\lim _{k \rightarrow \infty} \frac{1}{\left|B\left(x, r_{k}\right)\right|} \int_{B\left(x, r_{k}\right)}|b(x)-b(y)|\left|f_{h_{k}, l}(y)\right| d y=0 .
$$

It follows from (3.53)-(3.56) that (3.33) holds for almost every $x \in K$. Since $K \subset \subset \Omega$ is arbitrary, this gives the claim in $\Omega$.

Lemma 3.5. Let $f \in W^{1, p_{1}}(\Omega)$ and $b \in W^{1, p_{2}}(\Omega)$ with $1<p_{1}, p_{2}, p_{1} p_{2} /\left(p_{1}+\right.$ $\left.p_{2}\right)<\infty$. Let $h_{k}$ be positive real numbers so that $h_{k} \rightarrow 0$ and define the function $F_{b}(x, y): \Omega \times \Omega \rightarrow \mathbb{R}$ by $F_{b}(x, y)=|b(x)-b(y)|$. Assume that $|\Omega|<\infty$ and there exists $l \in\{1,2, \ldots, n\}$ such that $\delta(x) \leq \delta\left(x+h_{k} e_{l}\right)$ for almost every $x \in \Omega$ and all $k \geq 1$. Then, for almost every $x \in \Omega$, it holds that

$$
\begin{aligned}
& \lim _{k \rightarrow \infty} \int_{B(x, \delta(x))} \frac{F_{b}\left(x+h_{k} e_{l}, y+h_{k} e_{l}\right) f_{\tau\left(h_{k}\right), l}(y)-F_{b}(x, y) f(y)}{h_{k}} d y \\
& =\int_{B(x, \delta(x))}\left(D_{l, y} F_{b}(x, y)+D_{l, x} F_{b}(x, y)\right) f(y) d y+\int_{B(x, \delta(x))} F_{b}(x, y) D_{l} f(y) d y .
\end{aligned}
$$

Proof. Observe that

$$
\begin{aligned}
& \lim _{k \rightarrow \infty} \int_{B(x, \delta(x))} \frac{F_{b}\left(x+h_{k} e_{l}, y+h_{k} e_{l}\right) f_{\tau\left(h_{k}\right), l}(y)-F_{b}(x, y) f(y)}{h_{k}} d y \\
& =\lim _{k \rightarrow \infty} \int_{B(x, \delta(x))} F_{b}(x, y) f_{h_{k}, l}(y) d y+\lim _{k \rightarrow \infty} \int_{B(x, \delta(x))}\left(F_{x, b}\right)_{h_{k}, l}(y) f_{\tau\left(h_{k}\right), l}(y) d y \\
& \quad+\lim _{k \rightarrow \infty} \int_{B(x, \delta(x))}\left(F_{y+h_{k} e_{l}, b}\right)_{h_{k}, l}(x) f_{\tau\left(h_{k}\right), l}(y) d y,
\end{aligned}
$$

where

$$
\begin{aligned}
& \left(F_{x, b}\right)_{h_{k}, l}(y)=\frac{1}{h_{k}}\left(F_{b}\left(x, y+h_{k} e_{l}\right)-F_{b}(x, y)\right), \\
& \left(F_{y, b}\right)_{h_{k}, l}(x)=\frac{1}{h_{k}}\left(F_{b}\left(x+h_{k} e_{l}, y\right)-F_{b}(x, y)\right) .
\end{aligned}
$$

Note that $f_{h_{k}, l} \rightarrow D_{l} f$ in $L_{\text {loc }}^{p_{1}}(\Omega)$ and $F_{b}(x, \cdot) \in L^{p_{2}}(\Omega)$ for any $x \in \Omega$. It follows that $F_{b}(x, \cdot) f_{h_{k}, l} \rightarrow F_{b}(x, \cdot) D_{l} f$ in $L_{\text {loc }}^{p}(\Omega)$ by Hölder's inequality, which imply that

$$
\lim _{k \rightarrow \infty} \int_{B(x, \delta(x)-t)} F_{b}(x, y) f_{h_{k}, l}(y) d y=\int_{B(x, \delta(x)-t)} F_{b}(x, y) D_{l} f(y) d y,
$$

for all $x \in \Omega$ and a fixed $t \in(0, \delta(x)]$. On the other hand, since $\delta(x) \leq \delta\left(x+h_{k} e_{l}\right)$, we have that $B(x, \delta(x)) \cup B\left(x+h_{k} e_{l}, \delta(x)\right) \subset \Omega$. Moreover, for almost every $y \in \Omega$, we see that $y+h_{k} e_{l}$ is a Lebesgue point of $f$ for all $k \geq 1$. By [26, Lemma 2.8] we can get

$$
\left|f_{h_{k}, l}(y)\right| \leq C\left(M\left(|\nabla f| \chi_{\Omega}\right)(y)+M\left(|\nabla f| \chi_{\Omega}\right)\left(y+h_{k} e_{l}\right)\right)=: C \Gamma_{1}(y)
$$


for almost every $y \in B(x, \delta(x))$. By the $L^{p_{1}}$ bounds for $M$ and Minkowski's inequality, one can easily check that $\left\|\Gamma_{1}\right\|_{p_{1}} \leq C\|\nabla f\|_{p_{1}, \Omega}$. This together with the fact that $F_{b}(x, \cdot) \in L^{p_{2}}(\Omega)$, Hölder's inequality, (3.60) and the absolute continuity of the integral implies that for every $\epsilon>0$, there exists $t_{0}>0$ such that

$$
\begin{aligned}
& \left|\int_{B(x, \delta(x)) \backslash B(x, \delta(x)-t)} F_{b}(x, y) f_{h_{k}, l}(y) d y\right| \\
& \leq C|B(x, \delta(x))|^{1-1 / p}\left(\int_{B(x, \delta(x)) \backslash B(x, \delta(x)-t)}\left|F_{b}(x, y)\right|^{p_{2}} d y\right)^{1 / p_{2}} \\
& \quad \times\left(\int_{B(x, \delta(x)) \backslash B(x, \delta(x)-t)}\left|\Gamma_{1}(y)\right|^{p_{1}} d y\right)^{1 / p_{1}} \\
& \leq C\|\nabla f\|_{p_{1}, \Omega}|B(x, \delta(x))|^{1-1 / p}\left(\int_{B(x, \delta(x)) \backslash B(x, \delta(x)-t)}\left|F_{b}(x, y)\right|^{p_{2}} d y\right)^{1 / p_{2}} \\
& \leq C \epsilon,
\end{aligned}
$$

whenever $t \leq t_{0}$. Here the above constant $C>0$ is independent of $\epsilon$. Combining (3.61) with (3.59) yields that

$$
\lim _{k \rightarrow \infty} \int_{B(x, \delta(x))} F_{b}(x, y) f_{h_{k}, l}(y) d y=\int_{B(x, \delta(x))} F_{b}(x, y) D_{l} f(y) d y
$$

for almost every $x \in \Omega$.

Next, for every $\varepsilon>0$, there exists $t_{1}>0$ such that the same estimates as (3.61) hold for $\left(F_{x, b}\right)_{h_{k}, l}(y) f_{\tau\left(h_{k}\right), l}(y), D_{l, y} F_{b}(x, y) f(y),\left(F_{y+h_{k} e_{l}, b}\right)_{h_{k}, l}(x) f_{\tau\left(h_{k}\right), l}(y)$ and $D_{l, x} F_{b}(x, y) f(y)$, whenever $0<t \leq t_{1}$. So, we have only to show that for any fixed $0<t<\min \left\{\delta(x), t_{1}\right\}$ with $h_{k}<t$ for large $k \in \mathbb{N}$,

$$
\begin{aligned}
& \lim _{k \rightarrow \infty} \int_{B(x, \delta(x)-t)}\left(F_{x, b}\right)_{h_{k}, l}(y) f_{\tau\left(h_{k}\right), l}(y) d y=\int_{B(x, \delta(x)-t)} D_{l, y} F_{b}(x, y) f(y) d y \\
& \lim _{k \rightarrow \infty} \int_{B(x, \delta(x)-t)}\left(F_{y+h_{k} e_{l}, b}\right)_{h_{k}, l}(x) f_{\tau\left(h_{k}\right), l}(y) d y=\int_{B(x, \delta(x)-t)} D_{l, x} F_{b}(x, y) f(y) d y
\end{aligned}
$$

for almost every $x \in \Omega$.

Fix $x \in \Omega$. By the above analysis as in getting (3.60), we have that

$$
\begin{aligned}
\left|\left(F_{x, b}\right)_{h_{k}, l}(y)\right| & \leq\left|\frac{b\left(y+h_{k} e_{l}\right)-b(y)}{h_{k}}\right| \\
& \leq C\left(M\left(|\nabla b| \chi_{\Omega}\right)(y)+M\left(|\nabla b| \chi_{\Omega}\right)\left(y+h_{k} e_{l}\right)\right)=: C \Gamma_{2}(y)
\end{aligned}
$$

for almost every $y \in B(x, \delta(x))$. The fact that $|\nabla b| \in L^{p_{2}}(\Omega)$ together with the $L^{p_{2}}$ bounds for $M$ gives that $\left\|\Gamma_{2}\right\|_{p_{2}} \leq C\|\nabla b\|_{p_{2}, \Omega}$. This together with (3.60), (3.65) and Hölder's inequality implies that

$$
\begin{aligned}
& \int_{B(x, \delta(x)-t)}\left|\left(F_{x, b}\right)_{h_{k}, l}(y)\left(f_{\tau\left(h_{k}\right), l}(y)-f(y)\right)\right| d y \\
& \leq C\left|h_{k}\right| \int_{B(x, \delta(x))} \Gamma_{1}(y) \Gamma_{2}(y) d y \\
& \leq C\left|h _ { k } \| B ( x , \delta ( x ) ) | ^ { 1 - 1 / p } \| \Gamma _ { 1 } \| _ { p _ { 1 } } \| \Gamma _ { 2 } \left\|_{p_{2}} \leq C\left|h_{k}\left\|\left.B(x, \delta(x))\right|^{1-1 / p}\right\| \nabla f\left\|_{p_{1}, \Omega}\right\| \nabla b \|_{p_{2}, \Omega},\right.\right.\right.
\end{aligned}
$$


Sobolev boundedness and continuity for commutators of the local Hardy-Littlewood maximal function 225

for almost every $x \in \Omega$, which leads to

$$
\lim _{k \rightarrow \infty} \int_{B(x, \delta(x)-t)}\left|\left(F_{x, b}\right)_{h_{k}, l}(y)\left(f_{\tau\left(h_{k}\right), l}(y)-f(y)\right)\right| d y=0 .
$$

By the arguments similar to those used in deriving (3.62) we can prove that

$$
\lim _{k \rightarrow \infty} \int_{B(x, \delta(x)-t)}\left(F_{x, b}\right)_{h_{k}, l}(y) f(y) d y=\int_{B(x, \delta(x)-t)} D_{l, y} F_{b}(x, y) f(y) d y
$$

for all $x \in \Omega$. Equality (3.67) together with (3.66) leads to (3.63).

It remains to show (3.64). Since $b \in W^{1, p_{2}}(\Omega)$, it follows that for almost every $x \in \Omega, \lim _{k \rightarrow \infty} b_{h_{k}, l}(x)$ exists, and hence for such $x$, there exists $C_{x, b}>0$ such that $\left|b_{h_{k}, l}(x)\right| \leq C_{x, b}$. Therefore, we can get

$$
\begin{aligned}
\left|\left(F_{y+h_{k} e_{l}, b}\right)_{h_{k}, l}(x)\right| & \leq \frac{|| b\left(x+h_{k} e_{l}\right)-b\left(y+h_{k} e_{l}\right)|-| b(x)-b\left(y+h_{k} e_{l}\right)||}{h_{k}} \\
& \leq\left|b_{h_{k}, l}(x)\right| \leq C_{x, b}
\end{aligned}
$$

for every $y \in B(x, \delta(x))$. Combining (3.68) with (3.60) and Hölder's inequality yields that

$$
\begin{aligned}
& \int_{B(x, \delta(x))}\left|\left(F_{y+h_{k} e_{l}, b}\right) h_{h_{k}, l}(x)\left(f_{\tau\left(h_{k}\right), l}(y)-f(y)\right)\right| d y \\
& \leq C\left|h_{k}\right| \int_{B(x, \delta(x))} \Gamma_{1}(y) d y \\
& \leq C\left|h_{k}\right||B(x, \delta(x))|^{1-1 / p_{1}}\left\|\Gamma_{1}\right\|_{p_{1}} \leq C\left|h_{k}\right||B(x, \delta(x))|^{1-1 / p_{1}}\|\nabla f\|_{p_{1}, \Omega},
\end{aligned}
$$

which gives that

$$
\lim _{k \rightarrow \infty} \int_{B(x, \delta(x))}\left|\left(F_{y+h_{k} e_{l}, b}\right)_{h_{k}, l}(x)\left(f_{\tau\left(h_{k}\right), l}(y)-f(y)\right)\right| d y=0 .
$$

Hence, equality (3.64) reduces to the following

$$
\lim _{k \rightarrow \infty} \int_{B(x, \delta(x)-t)}\left(F_{y+h_{k} e_{l}, b}\right)_{h_{k}, l}(x) f(y) d y=\int_{B(x, \delta(x)-t)} D_{l, x} F_{b}(x, y) f(y) d y
$$

We can write

$$
\begin{aligned}
& \left|\lim _{k \rightarrow \infty} \int_{B(x, \delta(x)-t)}\left(F_{y+h_{k} e_{l}, b}\right)_{h_{k}, l}(x) f(y) d y-\int_{B(x, \delta(x)-t)} D_{l, x} F_{b}(x, y) f(y) d y\right| \\
& \leq \lim _{k \rightarrow \infty} \int_{B(x, \delta(x)-t)}\left|\left(F_{y+h_{k} e_{l}, b}\right)_{h_{k}, l}(x)-D_{l, x} F_{b}(x, y)\right| f(y) d y \\
& \leq \lim _{k \rightarrow \infty} \int_{B(x, \delta(x)-t)}\left|\left(F_{y+h_{k} e_{l}, b}\right)_{h_{k}, l}(x)-D_{l, x} F_{b}\left(x, y+h_{k} e_{l}\right)\right| f(y) d y \\
& \quad+\lim _{k \rightarrow \infty} \int_{B(x, \delta(x)-t)}\left|D_{l, x} F_{b}\left(x, y+h_{k} e_{l}\right)-D_{l, x} F_{b}(x, y)\right| f(y) d y .
\end{aligned}
$$

By the argument similar to that used in deriving (3.42) we have that

$$
\begin{aligned}
& \left|\int_{B(x, \delta(x)-t)}\right|\left(F_{y+h_{k} e_{l}, b}\right)_{h_{k}, l}(x)-D_{l, x} F_{b}\left(x, y+h_{k} e_{l}\right) \mid f(y) d y \\
& \leq|B(x, \delta(x)-t)|^{1-1 / p_{1}-1 / p_{2}}\|f\|_{p_{1}, \Omega}\left\|\left(F_{\cdot, b}\right)_{h_{k}, l}(x)-D_{l, x} F_{b}(x, \cdot)\right\|_{p_{2}, B(x, \delta(x))},
\end{aligned}
$$


because $B(x, \delta(x)-t)+h_{k} e_{l} \subset B\left(x, \delta(x)-t+h_{k}\right) \subset B(x, \delta(x))$. Similarly, it holds that

$$
\begin{aligned}
& \int_{B(x, \delta(x)-t)}\left|D_{l, x} F_{b}\left(x, y+h_{k} e_{l}\right)-D_{l, x} F_{b}(x, y)\right| f(y) d y \\
& \leq|B(x, \delta(x)-t)|^{1-1 / p}\|f\|_{p_{1}, \Omega}\left\|\left(D_{l, x} F_{b}\right)_{\tau\left(h_{k}\right), l}(x, \cdot)-D_{l, x} F_{b}(x, \cdot)\right\|_{p_{2}, B(x, \delta(x))} .
\end{aligned}
$$

Since $\overline{B(x, \delta(x))} \subset \subset \Omega$, we can see in the proof of Lemma 3.4 that

$$
\lim _{k \rightarrow \infty}\left\|\left(F_{\cdot, b}\right)_{h_{k}, l}(x)-D_{l, x} F_{b}(x, \cdot)\right\|_{p_{2}, B(x, \delta(x))}=0,
$$

which together with (3.72) yields that

$$
\lim _{k \rightarrow \infty} \int_{B(x, \delta(x)-t)}\left|\left(F_{y+h_{k} e_{l}, b}\right)_{h_{k}, l}(x)-D_{l, x} F_{b}\left(x, y+h_{k} e_{l}\right)\right| f(y) d y=0
$$

for almost every $x \in \Omega$. In the proof of Lemma 3.4 we also see that

$$
\left\|\left(D_{l, x} F_{b}\right)_{\tau\left(h_{k}\right), l}(x, \cdot)-D_{l, x} F_{b}(x, \cdot)\right\|_{p_{2}, B(x, \delta(x))} \rightarrow 0 \text { as } k \rightarrow \infty
$$

for all $x \in \Omega$. Combining (3.75) with (3.73) leads to

$$
\lim _{k \rightarrow \infty} \int_{B(x, \delta(x)-t)}\left|D_{l, x} F_{b}\left(x, y+h_{k} e_{l}\right)-D_{l, x} F_{b}(x, y)\right| f(y) d y=0
$$

for all $x \in \Omega$. It follows from (3.71), (3.74) and (3.76) that (3.70) holds for almost every $x \in \Omega$. Consequently, inequality (3.57) follows from (3.58) and (3.62)-(3.64).

By the arguments similar to those used to derive Lemma 3.5, we can get the following result. The details are omitted.

Lemma 3.6. Let $f \in W^{1, p_{1}}(\Omega)$ and $b \in W^{1, p_{2}}(\Omega)$ with $1<p_{1}, p_{2}, p_{1} p_{2} /\left(p_{1}+\right.$ $\left.p_{2}\right)<\infty$. Let $h_{k}$ be positive real numbers so that $h_{k} \rightarrow 0$ and define the function $F_{b}(x, y): \Omega \times \Omega \rightarrow \mathbb{R}$ by $F_{b}(x, y)=|b(x)-b(y)|$. Assume that $|\Omega|<\infty$ and there exists $l \in\{1,2, \ldots, n\}$ such that $\delta(x) \geq \delta\left(x+h_{k} e_{l}\right)$ for almost every $x \in \Omega$ and all $k \geq 1$. Then, for almost every $x \in \Omega$, we have

$$
\begin{aligned}
& \lim _{k \rightarrow \infty} \int_{B\left(x, \delta\left(x+h_{k} e_{l}\right)\right)} \frac{F_{b}\left(x+h_{k} e_{l}, y+h_{k} e_{l}\right) f_{\tau\left(h_{k}\right), l}(y)-F_{b}(x, y) f(y)}{h_{k}} d y \\
& =\int_{B(x, \delta(x))}\left(D_{l, y} F_{b}(x, y)+D_{l, x} F_{b}(x, y)\right) f(y) d y+\int_{B(x, \delta(x))} F_{b}(x, y) D_{l} f(y) d y .
\end{aligned}
$$

Lemma 3.7. [26, Lemma 2.11] Let $A_{j} \subset \mathbb{R}^{n}$ be measurable sets and let $h_{k} \in \mathbb{R}^{n}$ such that $\left|h_{k}\right| \rightarrow 0$ when $k \rightarrow \infty$. Then we can find a subsequence of $\left\{h_{k_{i}}\right\}$ such that for every $j$ and for almost every $x \in A_{j}$ we have $x+h_{k_{i}} \in A_{j}$ when $i$ is large enough.

Lemma 3.8. Let $1<p_{1}, p_{2}, p<\infty, 1 / p=1 / p_{1}+1 / p_{2}, f \in W^{1, p_{1}}(\Omega)$ and $b \in W^{1, p_{2}}(\Omega)$. Let $\left\{f_{j}\right\}_{j=1}^{\infty} \subsetneq W^{1, p_{1}}(\Omega)$ such that $f_{j} \rightarrow f$ in $W^{1, p_{1}}(\Omega)$ as $j \rightarrow \infty$. Assume that $|\Omega|<\infty$ and $K \subset \subset \Omega$. Then for all $l \in\{1,2, \ldots, n\}$, we have

$$
\lim _{j \rightarrow \infty}\left\|D_{l} M_{b, \Omega} f_{j}-D_{l} M_{b, \Omega} f\right\|_{p, K_{j}}=0
$$

where

$$
K_{j}:=\left\{x \in K: \delta(x) \in \mathcal{R}\left(f_{j}\right)(x) \cap \mathcal{R}(f)(x)\right\} .
$$


Proof. We may assume without loss of generality that all $f_{j} \geq 0$ and $f \geq 0$. Let us fix $l \in\{1,2, \ldots, n\}$. Since $M_{b, \Omega} f_{j}-M_{b, \Omega} f \in W^{1, p}(\Omega)$ for $p>1$, there exists a sequence $\left\{h_{k}\right\}_{k=1}^{\infty}, h_{k} \rightarrow 0^{+}$such that

$$
\begin{aligned}
& \lim _{k \rightarrow \infty} \frac{M_{b, \Omega} f_{j}\left(x+h_{k} e_{l}\right)-M_{b, \Omega} f_{j}(x)-\left(M_{b, \Omega} f\left(x+h_{k} e_{l}\right)-M_{b, \Omega} f(x)\right)}{h_{k}} \\
& =D_{l}\left(M_{b, \Omega} f_{j}-M_{b, \Omega} f\right)(x)
\end{aligned}
$$

for all $j \geq 1$ and almost every $x \in K$. By Lemma 3.7, there exists a subsequence $\left\{s_{k}\right\}_{k=1}^{\infty}$ of $\left\{h_{k}\right\}_{k=1}^{\infty}, s_{k} \rightarrow 0$ as $k \rightarrow \infty$ such that for almost every $x \in K_{j}$, we have that $x+s_{k} e_{l} \in K_{j}$ for all $j$ when $k$ is large enough. It is clear that $M_{b, \Omega} f_{j}(x)=u_{x, b, f_{j}}(\delta(x))$ and $M_{b, \Omega} f(x)=u_{x, b, f}(\delta(x))$. It follows that

$$
M_{b, \Omega} f_{j}(x)-M_{b, \Omega} f(x)=u_{x, b, f_{j}-f}(\delta(x)) .
$$

Similarly it holds that

$$
M_{b, \Omega} f_{j}\left(x+s_{k} e_{l}\right)-M_{b, \Omega} f\left(x+s_{k} e_{l}\right)=u_{x+s_{k} e_{l}, b, f_{j}-f}\left(\delta\left(x+s_{k} e_{l}\right)\right) .
$$

Combining (3.80) with (3.78) and (3.79) implies that

$$
\begin{aligned}
& \left|D_{l} M_{b, \Omega} f_{j}(x)-D_{l} M_{b, \Omega} f(x)\right| \\
& \leq\left|\lim _{k \rightarrow \infty} \frac{u_{x+s_{k} e_{l}, b, f_{j}-f}\left(\delta\left(x+s_{k} e_{l}\right)\right)-u_{x, b, f_{j}-f}(\delta(x))}{s_{k}}\right|
\end{aligned}
$$

for almost every $x \in K_{j}$. The continuity of $u_{x, b, f_{j}-f}(r)$ yield that for almost every $x \in \Omega$, there exists a sequence of numbers $\left\{r_{\ell}\right\}_{\ell=1}^{\infty}, r_{\ell}>0, r_{\ell} \rightarrow 1$ as $\ell \rightarrow \infty$ such that

$$
u_{x, b, f_{j}-f}(\delta(x))=\lim _{\ell \rightarrow \infty} u_{x, b, f_{j}-f}\left(r_{\ell} \delta(x)\right)=\lim _{\ell \rightarrow \infty} A_{r_{\ell}, b, f_{j}-f}(x) .
$$

Similarly we can get

$$
u_{x+s_{k} e_{l}, b, f_{j}-f}\left(\delta\left(x+s_{k} e_{l}\right)\right)=\lim _{\ell \rightarrow \infty} A_{r_{\ell}, b, f_{j}-f}\left(x+s_{k} e_{l}\right)
$$

for almost every $x \in \Omega$ and all $k \geq 1$. Invoking Lemma 3.1 we have $A_{r_{\ell}, b, f_{j}-f} \in$ $W^{1, p}(\Omega)$. Therefore, there exists a subsequence $\left\{\iota_{k}\right\}_{k=1}^{\infty}$ of $\left\{s_{k}\right\}_{k=1}^{\infty}, \iota_{k} \rightarrow 0^{+}$such that

$$
\frac{A_{r_{\ell}, b, f_{j}-f}\left(x+\iota_{k} e_{l}\right)-A_{r_{\ell}, b, f_{j}-f}(x)}{\iota_{k}} \rightarrow D_{l} A_{r_{\ell}, b, f_{j}-f}(x) \text { as } k \rightarrow \infty
$$

for all $j, \ell \geq 1$ and almost every $x \in K$. By (3.82)-(3.84) and Lemma 3.1, one has

$$
\begin{aligned}
& \left|D_{l} M_{b, \Omega} f_{j}(x)-D_{l} M_{b, \Omega} f(x)\right| \\
& \leq\left|\lim _{k \rightarrow \infty} \lim _{\ell \rightarrow \infty} \frac{A_{r_{\ell}, b, f_{j}-f}\left(x+\iota_{k} e_{l}\right)-A_{r_{\ell}, b, f_{j}-f}(x)}{\iota_{k}}\right| \\
& \leq \lim _{\ell \rightarrow \infty}\left|\lim _{k \rightarrow \infty} \frac{A_{r_{\ell}, b, f_{j}-f}\left(x+\iota_{k} e_{l}\right)-A_{r_{\ell}, b, f_{j}-f}(x)}{\iota_{k}}\right| \\
& \leq \lim _{\ell \rightarrow \infty}\left|D_{l} A_{r_{\ell}, b, f_{j}-f}(x)\right| \\
& \leq 2\left(M_{b, \Omega}\left|\nabla\left(f_{j}-f\right)\right|(x)+M_{\Omega}\left(|\nabla b|\left(f_{j}-f\right)\right)(x)\right)+|\nabla b|(x) M_{\Omega}\left|f_{j}-f\right|(x)
\end{aligned}
$$

for almost every $x \in K_{j}$. Combining (3.85) with (1.4), the $L^{p}$ bounds for $M_{\Omega}$ and Hölder's inequality yields (3.77). 
Lemma 3.9. [26, Corollary 2.7] Let $1<p<\infty$ and $A$ be a measurable subset of $\Omega$. Let $f_{j}$ be a sequence in $W_{\mathrm{loc}}^{1,1}(\Omega)$ so that $f_{j}$ converges to zero in the sense of distributions:

$$
\int_{\Omega} f_{j}(x) \varphi(x) d x \rightarrow 0 \text { as } j \rightarrow \infty \quad \text { for every } \varphi \in \mathcal{C}_{0}^{\infty}(\Omega) .
$$

Suppose that $\left|\nabla f_{j}(x)\right| \leq F(x)+F_{j}(x)$ for almost every $x \in \Omega$ and $\|F\|_{p, \Omega}<\infty$ and $\left\|F_{j}\right\|_{p, \Omega} \rightarrow 0$ as $j \rightarrow \infty$. Suppose also that for all $\epsilon>0$ and $1 \leq l \leq n$, it holds that $\left|\left\{x \in A: D_{l} f_{j}(x)>\epsilon\right\}\right| \rightarrow 0$ as $j \rightarrow \infty$ or $\left|\left\{x \in A: D_{l} f_{j}(x)<-\epsilon\right\}\right| \rightarrow 0$ as $j \rightarrow \infty$. Then

$$
\lim _{j \rightarrow \infty}\left\|D_{l} f_{j}\right\|_{p, A}=0 \text {. }
$$

3.2. Proof of Theorem 1.2. We will divide the proof of Theorem 1.2 into two steps:

Step 1: Proofs of (1.7) and (1.8). Let $\left\{t_{k}\right\}_{k \geq 1}$ be an enumeration of the rationals between 0 and 1 . For $k \geq 1$, we define the function $g_{k}: \Omega \rightarrow[-\infty, \infty]$ by $g_{k}(x)=$ $\max _{1 \leq j \leq k} A_{t_{j}, b, f}(x)$. One can easily check that $g_{k} \rightarrow M_{b, \Omega} f$ pointwise as $k \rightarrow \infty$. Moreover, $\left\{g_{k}\right\}_{k=1}^{\infty}$ is an increasing sequence of functions in $W^{1, p}(\Omega)$ and

$$
\begin{aligned}
\left|\nabla g_{k}(x)\right| & =\left|\nabla \max _{1 \leq j \leq k} A_{t_{j}, b, f}(x)\right| \leq \max _{1 \leq j \leq k}\left|\nabla A_{t_{j}, b, f}(x)\right| \\
& \leq 2\left(M_{b, \Omega}|\nabla f|(x)+M_{\Omega}(|\nabla b| f)(x)\right)+|\nabla b|(x) M_{\Omega} f(x),
\end{aligned}
$$

for almost every $x \in \Omega$. Moreover, $g_{k}(x) \leq M_{b, \Omega} f(x)$ for every $x \in \Omega$. This together with (3.86), (1.4), the $L^{p}$ bounds for $M_{\Omega}$ and Hölder's inequality implies that

$$
\left\|g_{k}\right\|_{1, p, \Omega}=\left\|g_{k}\right\|_{p, \Omega}+\left\|\nabla g_{k}\right\|_{p, \Omega} \leq C_{p_{1}, p_{2}}\|b\|_{1, p_{2}, \Omega}\|f\|_{1, p_{1}, \Omega},
$$

which implies that $\left\{g_{k}\right\}_{k=1}^{\infty}$ is a bounded sequence in $W^{1, p}(\Omega)$ such that $g_{k} \rightarrow M_{b, \Omega} f$ almost everywhere in $\Omega$ as $k \rightarrow \infty$. A weak compactness argument shows that $M_{b, \Omega} f \in W^{1, p}(\Omega)$ and

$$
g_{k} \rightarrow M_{b, \Omega} f \text { and } \nabla g_{k} \rightarrow \nabla M_{b, \Omega} f \text { weakly in } L^{p}(\Omega) \text { as } k \rightarrow \infty .
$$

Applying Proposition 2.1 to (3.86) with $a_{k}=\left|\nabla g_{k}\right|$ and

$$
b_{k}=2\left(M_{b, \Omega}|\nabla f|+M_{\Omega}(|\nabla b| f)\right)+|\nabla b|(x) M_{\Omega} f,
$$

we can get (1.7). By (1.7), (1.4), the bounds for $M_{\Omega}$ and Hölder's inequality, we now obtain that

$$
\begin{aligned}
& \left\|M_{b, \Omega} f\right\|_{1, p, \Omega} \\
& =\left\|M_{b, \Omega} f\right\|_{p, \Omega}+\left\|\nabla M_{b, \Omega} f\right\|_{p, \Omega} \\
& \leq C_{p_{1}, p_{2}}\|b\|_{p_{2}, \Omega}\|f\|_{p_{1}, \Omega}+2\left\|M_{b, \Omega}|\nabla f|\right\|_{p, \Omega}+2\left\|M_{\Omega}(|\nabla b| f)\right\|_{p, \Omega}+\left\||\nabla b| M_{\Omega} f\right\|_{p, \Omega} \\
& \leq C_{p_{1}, p_{2}}\|b\|_{1, p_{2}, \Omega}\|f\|_{1, p_{1}, \Omega},
\end{aligned}
$$

which gives (1.8).

Step 2: Proof of the continuity part. Let $|\Omega|<\infty$ and $1<p_{1}, p_{2}, p_{1} p_{2} /\left(p_{1}+p_{2}\right)<$ $\infty$. Let $f \in W^{1, p_{1}}(\Omega), b \in W^{1, p_{2}}(\Omega)$ and $\left\{f_{j}\right\}_{j=1}^{\infty}$ be a sequence of functions in $W^{1, p_{1}}(\Omega)$ such that $f_{j} \rightarrow f$ in $W^{1, p_{1}}(\Omega)$ as $j \rightarrow \infty$. Without loss of generality we may assume that all $f_{j} \geq 0$ and $f \geq 0$. We want to show that

$$
\left\|M_{b, \Omega} f_{j}-M_{b, \Omega} f\right\|_{1, p, \Omega} \rightarrow 0 \text { as } j \rightarrow \infty .
$$


Sobolev boundedness and continuity for commutators of the local Hardy-Littlewood maximal function 229

By (1.4) we known that $M_{b, \Omega} f_{j} \rightarrow M_{b, \Omega} f$ in $L^{p}\left(\mathbb{R}^{n}\right)$ as $j \rightarrow \infty$. Thus, to conclude (3.87), it suffices to show that

$$
\left\|D_{l} M_{b, \Omega} f_{j}-D_{l} M_{b, \Omega} f\right\|_{p, \Omega} \rightarrow 0 \text { when } j \rightarrow \infty
$$

for any $l=1,2, \ldots, n$.

We only work with (3.88) for $l=n$ and the other cases are analogous. For convenience, we set

$$
\begin{gathered}
G(x):=4\left(M_{b, \Omega}|\nabla f|(x)+M_{\Omega}(|\nabla b| f)(x)\right)+2|\nabla b|(x) M_{\Omega} f(x), \\
F_{j}(x):=2\left(M_{b, \Omega}\left|\nabla\left(f_{j}-f\right)\right|(x)+M_{\Omega}\left(|\nabla b|\left(f_{j}-f\right)\right)(x)\right)+|\nabla b|(x) M_{\Omega}\left|f_{j}-f\right|(x) .
\end{gathered}
$$

By (1.7) and the sublinearity of $M_{b, \Omega}$ and $M_{\Omega}$ we have

$$
\begin{aligned}
& \left|\nabla\left(M_{b, \Omega} f_{j}-M_{b, \Omega} f\right)(x)\right| \\
& \leq 4\left(M_{b, \Omega}|\nabla f|(x)+M_{\Omega}(|\nabla b| f)(x)\right)+2|\nabla b|(x) M_{\Omega} f(x) \\
& \quad+2\left(M_{b, \Omega}\left|\nabla\left(f_{j}-f\right)\right|(x)+M_{\Omega}\left(|\nabla b|\left(f_{j}-f\right)\right)(x)\right)+|\nabla b|(x) M_{\Omega}\left|f_{j}-f\right|(x) \\
& \leq G(x)+F_{j}(x)
\end{aligned}
$$

for almost every $x \in \Omega$. One can easily check that $G(\cdot) \in L^{p}(\Omega)$ and

$$
\begin{aligned}
\left\|F_{j}\right\|_{p, \Omega} & \leq 2\left\|M_{b, \Omega}\left|\nabla\left(f_{j}-f\right)\right|\right\|_{p, \Omega}+2\left\|M_{\Omega}\left(|\nabla b|\left(f_{j}-f\right)\right)\right\|_{p, \Omega}+\left\||\nabla b| M_{\Omega}\left|f_{j}-f\right|\right\|_{p, \Omega} \\
& \leq C_{p_{1}, p_{2}, n}\|b\|_{1, p_{2}, \Omega}\left\|f_{j}-f\right\|_{1, p_{1}, \Omega} .
\end{aligned}
$$

Hence, for a fixed $\epsilon>0$, there exists $N_{0} \in \mathbb{N} \backslash\{0\}$ such that $\left\|F_{j}\right\|_{p, \Omega}<\epsilon$ for all $j \geq N_{0}$. Moreover, there exists $K \subset \subset \Omega$ such that $\|G\|_{p, \Omega \backslash K}<\epsilon$. By the absolute continuity, there exists $\eta>0$ such that $\|G\|_{p, A}<\epsilon$ whenever $A$ is a measurable set with $A \subset K$ and $|A|<\eta$. Therefore, we get from (3.89) that

$$
\left\|D_{n}\left(M_{b, \Omega} f_{j}-M_{b, \Omega} f\right)\right\|_{p, \Omega \backslash K} \leq\|G\|_{p, \Omega \backslash K}+\left\|F_{j}\right\|_{p, \Omega} \leq 2 \epsilon
$$

for any $j \geq N_{0}$. It follows that

$$
\left\|D_{n}\left(M_{b, \Omega} f_{j}-M_{b, \Omega} f\right)\right\|_{p, \Omega \backslash K} \rightarrow 0 \text { as } j \rightarrow \infty .
$$

Hence, to prove (3.88) for $l=n$, it is enough to show that

$$
\left\|D_{n}\left(M_{b, \Omega} f_{j}-M_{b, \Omega} f\right)\right\|_{p, K} \rightarrow 0 \text { as } j \rightarrow \infty .
$$

Set

$$
H=\{x \in K: \delta(x) \notin \mathcal{R}(f)(x)\} .
$$

Then proving (3.91) reduces to proving that

$$
\left\|D_{n}\left(M_{b, \Omega} f_{j}-M_{b, \Omega} f\right)\right\|_{p, H} \rightarrow 0 \text { as } j \rightarrow \infty,
$$

and

$$
\left\|D_{n}\left(M_{b, \Omega} f_{j}-M_{b, \Omega} f\right)\right\|_{p, K \backslash H} \rightarrow 0 \text { as } j \rightarrow \infty .
$$

We now prove (3.92). By the compactness of $\mathcal{R}(f)(x)$, there exists a constant $\gamma>0$ such that

$$
|\{x \in H: \mathcal{R}(f)(x) \nsubseteq[0, \delta(x)-\gamma]\}|=:\left|A_{\gamma}\right|<\frac{\eta}{4} .
$$

For convenience, we define the functions $\mathcal{A}_{x, b, f}(r):[0, \delta(x)] \rightarrow \mathbb{R}$ by

$$
\mathcal{A}_{x, b, f}(0)=0 \text {, }
$$




$$
\begin{aligned}
\mathcal{A}_{x, b, f}(r)= & \frac{1}{|B(x, r)|} \int_{B(x, r)}\left(D_{n, y}(|b(x)-b(y)|)+D_{n, x}(|b(x)-b(y)|)\right) f(y) d y \\
& +\frac{1}{|B(x, r)|} \int_{B(x, r)}|b(x)-b(y)| D_{n} f(y) d y .
\end{aligned}
$$

It was observed that $\mathcal{A}_{x, b, f}$ are continuous on $(0, \delta(x)]$ for all $x \in \Omega$ and at $r=0$ for almost every $x \in \Omega$. Hence, for almost every $x \in \Omega$, the function $\mathcal{A}_{x, b, f}$ is uniformly continuous on $[0, \delta(x)]$. Further we can find a constant $\gamma(x) \in(0, \gamma)$ such that

$$
\left|\mathcal{A}_{x, b, f}\left(r_{1}\right)-\mathcal{A}_{x, b, f}\left(r_{2}\right)\right|<\epsilon \text { whenever }\left|r_{1}-r_{2}\right|<\gamma(x) .
$$

Write

$$
K=\left(\bigcup_{k=1}^{\infty}\left\{x \in K ; k^{-1}<\gamma(x)<\gamma\right\}\right) \bigcup \mathcal{N},
$$

where $|\mathcal{N}|=0$. Therefore, there exists a constant $\beta \in(0, \gamma)$ such that

$$
\begin{aligned}
& \mid\left\{x \in K:\left|\mathcal{A}_{x, b, f}\left(r_{1}\right)-\mathcal{A}_{x, b, f}\left(r_{2}\right)\right| \geq \epsilon \text { for some } r_{1}, r_{2} \text { with }\left|r_{1}-r_{2}\right|<\beta\right\} \mid \\
& =:\left|A_{\beta}\right|<\frac{\eta}{4} .
\end{aligned}
$$

By Lemma 3.2, there exists $N_{1} \in \mathbb{N} \backslash\{0\}$ such that

$$
\left|\left\{x \in K ; \mathcal{R}\left(f_{j}\right)(x) \nsubseteq \mathcal{R}(f)(x)_{(\beta)}\right\}\right|=:\left|K^{j}\right|<\frac{\eta}{4} \text { when } j \geq N_{1} .
$$

Invoking Lemma 3.4, for almost every $x \in \Omega$, any $r_{1} \in \mathcal{R}\left(f_{j}\right)(x)$ and $r_{2} \in \mathcal{R}(f)(x)$ with $r_{1}, r_{2}<\delta(x)$, we have

$$
\begin{aligned}
& \left|D_{n}\left(M_{b, \Omega} f_{j}-M_{b, \Omega} f\right)(x)\right|=\left|\mathcal{A}_{x, b, f_{j}}\left(r_{1}\right)-\mathcal{A}_{x, b, f}\left(r_{2}\right)\right| \\
& \leq\left|\mathcal{A}_{x, b, f_{j}}\left(r_{1}\right)-\mathcal{A}_{x, b, f}\left(r_{1}\right)\right|+\left|\mathcal{A}_{x, b, f}\left(r_{1}\right)-\mathcal{A}_{x, b, f}\left(r_{2}\right)\right| .
\end{aligned}
$$

When $r_{1}=0$, it is easy to see that $\left|\mathcal{A}_{x, b, f_{j}}\left(r_{1}\right)-\mathcal{A}_{x, b, f}\left(r_{1}\right)\right|=0$. When $r_{1}>0$, it was noted that

$$
\begin{aligned}
\left|\mathcal{A}_{x, b, f}\left(r_{1}\right)\right| \leq & \frac{1}{|B(x, r)|} \int_{B(x, r)}\left|D_{n, y}(|b(x)-b(y)|)\right| f(y) d y \\
& +\frac{1}{|B(x, r)|} \int_{B(x, r)}\left|D_{n, x}(|b(x)-b(y)|)\right| f(y) d y \\
& +\frac{1}{|B(x, r)|} \int_{B(x, r)}|b(x)-b(y)|\left|D_{n} f(y)\right| d y \\
\leq & M_{\Omega}\left(\left|D_{n} b\right| f\right)(x)+\left|D_{n} b(x)\right| M_{\Omega} f(x)+M_{b, \Omega}\left|D_{n} f\right|(x)=: \hbar(f)(x) .
\end{aligned}
$$

Combining (3.97) with (3.98) yields that for almost every $x \in \Omega$, it holds that

$$
\left|D_{n}\left(M_{b, \Omega} f_{j}-M_{b, \Omega} f\right)(x)\right| \leq \hbar\left(f_{j}-f\right)(x)+\left|\mathcal{A}_{x, b, f}\left(r_{1}\right)-\mathcal{A}_{x, b, f}\left(r_{2}\right)\right|
$$

for any $r_{1} \in \mathcal{R}\left(f_{j}\right)(x)$ and $r_{2} \in \mathcal{R}(f)(x)$ with $r_{1}, r_{2}<\delta(x)$.

On the other hand, it holds that

$$
\begin{aligned}
& \left\|\hbar\left(f_{j}-f\right)\right\|_{p, \Omega} \\
& \leq\left\|M_{\Omega}\left(\left|D_{n} b\right|\left(f_{j}-f\right)\right)\right\|_{p, \Omega}+\left\|D_{n} b M_{\Omega}\left(f_{j}-f\right)\right\|_{p, \Omega}+\left\|M_{b, \Omega}\left(D_{n}\left(f_{j}-f\right)\right)\right\|_{p, \Omega} \\
& \leq C_{p_{1}, p_{2}, n}\left(\left\|D_{n} b\right\|_{p_{2}, \Omega}\left\|f_{j}-f\right\|_{p_{1}, \Omega}+\left\|D_{n} b\right\|_{p_{2}, \Omega} \| f_{j}\right. \\
& \left.\quad-f\left\|_{p_{1}, \Omega}+\right\| b\left\|_{p_{2}, \Omega}\right\| D_{n} f_{j}-D_{n} f \|_{p_{1}, \Omega}\right) \\
& \leq C_{p_{1}, p_{2}, n}\|b\|_{1, p_{2}, \Omega}\|f\|_{1, p_{1}, \Omega} .
\end{aligned}
$$


Sobolev boundedness and continuity for commutators of the local Hardy-Littlewood maximal function 231

Consequently, there exists $N_{2} \in \mathbb{N} \backslash\{0\}$ such that

$$
\left\|\hbar\left(f_{j}-f\right)\right\|_{p, \Omega}<\epsilon, \text { for all } j \geq N_{2} .
$$

Observe that for any $r_{1} \in \mathcal{R}\left(f_{j}\right)(x)$ and $r_{2} \in \mathcal{R}(f)(x)$ with $r_{1}, r_{2}<\delta(x)$, we get from (3.98) that

$$
\left|\mathcal{A}_{x, b, f}\left(r_{1}\right)-\mathcal{A}_{x, b, f}\left(r_{2}\right)\right| \leq 2 \hbar(f)(x) \leq G(x) .
$$

If $x \in H \backslash\left(A_{\gamma} \cup A_{\beta} \cup K^{j}\right)$ we can choose $r_{1} \in \mathcal{R}\left(f_{j}\right)(x)$ and $r_{2} \in \mathcal{R}(f)(x)$ such that $r_{1}, r_{2}<\delta(x),\left|r_{1}-r_{2}\right|<\beta$ and

$$
\left|\mathcal{A}_{x, b, f}\left(r_{1}\right)-\mathcal{A}_{x, b, f}\left(r_{2}\right)\right|<\epsilon .
$$

Observe from (3.94)-(3.96) that $\left|A_{\gamma} \cup A_{\beta} \cup K^{j}\right|<\eta$ for all $j \geq N_{1}$. It follows from (3.99)-(3.102) that

$$
\begin{aligned}
\left\|D_{n}\left(M_{b, \Omega} f_{j}-M_{b, \Omega} f\right)\right\|_{p, H} & \leq\left\|\hbar\left(f_{j}-f\right)\right\|_{p, \Omega}+\|\epsilon\|_{p, H \backslash\left(A_{\gamma} \cup A_{\beta} \cup K^{j}\right)}+\|G\|_{p, A_{\gamma} \cup A_{\beta} \cup K^{j}} \\
& \leq(2+|K|) \epsilon,
\end{aligned}
$$

for all $j \geq \max \left\{N_{1}, N_{2}\right\}$, which proves (3.92).

It remains to prove (3.93). Let $\left\{h_{k}\right\}_{k=1}^{\infty}$ be a sequence of numbers such that $h_{k} \rightarrow 0^{+}$as $k \rightarrow \infty$. Following the notations in [26], we set

$$
\begin{aligned}
& E^{j}:=\left\{x \in K \backslash H: \delta(x) \in \mathcal{R}\left(f_{j}\right)(x)\right\}, \\
& E^{+}:=\left\{x \in K \backslash H: \delta\left(x+h_{k} e_{n}\right) \geq \delta(x) \text { for infinitely many } k\right\}, \\
& E^{-}:=\left\{x \in K \backslash H: \delta\left(x+h_{k} e_{n}\right) \leq \delta(x) \text { for infinitely many } k\right\} .
\end{aligned}
$$

Note that $K \backslash H \subset E^{j} \cup E^{+} \cup E^{-}$. Hence, proving (3.93) reduces to proving the following

$$
\begin{aligned}
& \left\|D_{n}\left(M_{b, \Omega} f_{j}-M_{b, \Omega} f\right)\right\|_{p, E^{j}} \rightarrow 0 \text { as } j \rightarrow \infty, \\
& \left\|D_{n}\left(M_{b, \Omega} f_{j}-M_{b, \Omega} f\right)\right\|_{p, E^{+}} \rightarrow 0 \text { as } j \rightarrow \infty, \\
& \left\|D_{n}\left(M_{b, \Omega} f_{j}-M_{b, \Omega} f\right)\right\|_{p, E^{-}} \rightarrow 0 \text { as } j \rightarrow \infty .
\end{aligned}
$$

An application of Lemma 3.7 leads to (3.103). We now prove (3.104) and (3.105). We first prove (3.104). By the definition of $E^{+}$, it holds that $\delta(x) \leq \delta\left(x+h_{k} e_{n}\right)$ for infinitely many $k$ if $x \in E^{+}$. In order to apply Lemma 3.5, without loss of generality we may assume that $\delta(x) \leq \delta\left(x+h_{k} e_{n}\right)$ for all $k \geq K_{0}$ by extracting a subsequence if $x \in E^{+}$, where $K_{0}$ is a large positive number. Moreover, for almost every $x \in E^{+}$, we have that $x+h_{k} e_{n} \in E^{+}$for $k \geq K_{0}$. Hence, for almost every $x \in E^{+}$and $k \geq K_{0}$, we have

$$
M_{b, \Omega} f\left(x+h_{k} e_{n}\right) \geq u_{x+h_{k} e_{n}, b, f}(\delta(x))
$$

and

$$
M_{b, \Omega} f(x)=u_{x, b, f}(\delta(x)) .
$$

These above inequalities together with Lemma 3.5 will lead to

$$
\begin{aligned}
D_{n} M_{b, \Omega} f(x) & =\lim _{k \rightarrow \infty} \frac{1}{h_{k}}\left(M_{b, \Omega} f\left(x+h_{k} e_{n}\right)-M_{b, \Omega} f(x)\right) \\
& \geq \limsup _{k \rightarrow \infty} \frac{1}{h_{k}}\left(u_{x+h_{k} e_{n}, b, f}(\delta(x))-u_{x, b, f}(\delta(x))\right)=\mathcal{A}_{x, b, f}(\delta(x))
\end{aligned}
$$

for almost every $x \in E^{+}$. This combined with Lemma 3.4 implies that

$$
D_{n} M_{b, \Omega} f(x) \geq \mathcal{A}_{x, b, f}(r)
$$


for all $r \in \mathcal{R}(f)(x)$ (equality if $r<\delta(x)$ ). By the definitions of $\beta$ and $K^{j}$ we see that $\mathcal{R}\left(f_{j}\right)(x) \subset \mathcal{R}(f)(x)_{(\beta)}$ when $j \geq N_{1}$ and $x \in \Omega \backslash K^{j}$. Hence, for every $x \in E^{+} \backslash\left(K^{j} \cup E^{j}\right)$ with $j \geq N_{1}$, there exists $r_{j} \in \mathcal{R}\left(f_{j}\right)(x), r_{j}<\delta(x)$ such that $\left|r_{j}-r\right| \leq \beta$ for some $r \in \mathcal{R}(f)(x)$ (Here $r$ may be $\delta(x)$ ). Note that $r_{j}<\delta(x)$ since $x \in E^{+} \backslash\left(K^{j} \cup E^{j}\right)$. By Lemma 3.4 and (3.106), we have that for almost every $x \in E^{+} \backslash\left(K^{j} \cup E^{j}\right)$ with $j \geq N_{1}$, it holds that

$$
\begin{aligned}
D_{n} M_{b, \Omega} f(x)-D_{n} M_{b, \Omega} f_{j}(x) & \geq \mathcal{A}_{x, b, f}(r)-\mathcal{A}_{x, b, f_{j}}\left(r_{j}\right) \\
& \geq \mathcal{A}_{x, b, f}(r)-\mathcal{A}_{x, b, f}\left(r_{j}\right)+\mathcal{A}_{x, b, f}\left(r_{j}\right)-\mathcal{A}_{x, b, f_{j}}\left(r_{j}\right) \\
& =\mathcal{A}_{x, b, f}(r)-\mathcal{A}_{x, b, f}\left(r_{j}\right)+\mathcal{A}_{x, b, f-f_{j}}\left(r_{j}\right) .
\end{aligned}
$$

By the continuity of the functions $\mathcal{A}_{x, b, f}$ on $[0, \delta(x)]$ we note that

$$
\left|\left\{x \in \Omega:\left|\mathcal{A}_{x, b, f}(r)-\mathcal{A}_{x, b, f}\left(r_{j}\right)\right| \geq \epsilon / 2\right\}\right| \rightarrow 0 \text { as } j \rightarrow \infty .
$$

By (3.98) we see that

$$
\left|\mathcal{A}_{x, b, f-f_{j}}\left(r_{j}\right)\right| \leq \hbar\left(f_{j}-f\right)(x),
$$

where $\hbar$ is given as in (3.98). Note that $\left\|\hbar\left(f_{j}-f\right)\right\|_{p, \Omega} \rightarrow 0$ as $j \rightarrow \infty$. This yields that

$$
\left|\left\{x \in \Omega:\left|\mathcal{A}_{x, b, f-f_{j}}\left(r_{j}\right)\right| \geq \epsilon / 2\right\}\right| \rightarrow 0 \text { as } j \rightarrow \infty .
$$

It follows from (3.107)-(3.109) that

$$
\begin{aligned}
& \left|\left\{x \in E^{+} \backslash\left(K^{j} \cup E^{j}\right): D_{n} M_{b, \Omega} f(x)-D_{n} M_{b, \Omega} f_{j}(x) \leq-\epsilon\right\}\right| \\
& \leq\left|\left\{x \in E^{+} \backslash\left(K^{j} \cup E^{j}\right): \mathcal{A}_{x, b, f}(r)-\mathcal{A}_{x, b, f}\left(r_{j}\right)+\mathcal{A}_{x, b, f-f_{j}}\left(r_{j}\right) \leq-\epsilon\right\}\right| \rightarrow 0
\end{aligned}
$$

as $j \rightarrow \infty$. By (3.89), (3.110) and Lemma 3.9 we have

$$
\left\|D_{n} M_{b, \Omega} f_{j}-D_{n} M_{b, \Omega} f\right\|_{p, E^{+} \backslash\left(K^{j} \cup E^{j}\right)} \rightarrow 0 \text { as } j \rightarrow \infty .
$$

On the other hand, by (3.95) and (3.100) one can get

$$
\left\|D_{n} M_{b, \Omega} f_{j}-D_{n} M_{b, \Omega} f\right\|_{p, E^{+} \cap K^{j}} \leq\left\|\hbar\left(f_{j}-f\right)\right\|_{p, \Omega}+\|G\|_{p, K^{j}} \leq 3 \epsilon
$$

for any $j \geq \max \left\{N_{1}, N_{2}\right\}$, which leads to

$$
\left\|D_{n} M_{b, \Omega} f_{j}-D_{n} M_{b, \Omega} f\right\|_{p, E^{+} \cap K^{j}} \rightarrow 0 \text { as } j \rightarrow \infty .
$$

Then (3.104) follows from (3.103), (3.111) and (3.113).

Now we prove (3.105). This proof is similar to that of (3.104). We may assume that $x+h_{k} e_{n} \in E^{-}$for almost every $x \in E^{-}$when $k \geq K_{1}$ for a large $K_{1}>0$. It follows that

$$
M_{b, \Omega} f\left(x+h_{k} e_{n}\right)=u_{x+h_{k} e_{n}, b, f}\left(\delta\left(x+h_{k} e_{n}\right)\right)
$$

and

$$
M_{b, \Omega} f(x)=u_{x, b, f}(\delta(x)) \geq u_{x, b, f}\left(\delta\left(x+h_{k} e_{n}\right)\right)
$$

for almost every $x \in E^{-}$and $k$ large enough. Similar arguments to those in deriving (3.106) together with Lemma 3.6 give that

$$
D_{n} M_{b, \Omega} f(x) \leq \mathcal{A}_{x, b, f}(r)
$$

for all $r \in \mathcal{R}(f)(x)$ (equality if $r<\delta(x)$ ). The definitions of $\beta$ and $E^{j}$ imply that $\mathcal{R}\left(f_{j}\right)(x) \subset \mathcal{R}(f)(x)_{(\beta)}$ when $j \geq N_{1}$ and $x \in \Omega \backslash K^{j}$. It follows that for every $x \in E^{-} \backslash K^{j}$ with $j \geq N_{1}$, there exists $r_{j} \in \mathcal{R}\left(f_{j}\right)(x), r_{j}<\delta(x)$ such that $\left|r_{j}-r\right| \leq \beta$ 
for some $r \in \mathcal{R}(f)(x)$ (Here $r$ may be $\delta(x)$ ). By the arguments similar to those used in getting (3.107), one has

$$
\begin{aligned}
D_{n} M_{b, \Omega} f(x)-D_{n} M_{b, \Omega} f_{j}(x) & \leq \mathcal{A}_{x, b, f}(r)-\mathcal{A}_{x, b, f_{j}}\left(r_{j}\right) \\
& \leq \mathcal{A}_{x, b, f}(r)-\mathcal{A}_{x, b, f}\left(r_{j}\right)+\mathcal{A}_{x, b, f-f_{j}}\left(r_{j}\right)
\end{aligned}
$$

for almost every $x \in E^{-} \backslash\left(K^{j} \cup E^{j}\right)$ with $j \geq N_{1}$. It follows from (3.115), (3.108) and (3.109) that

$$
\begin{aligned}
& \left|\left\{x \in E^{-} \backslash\left(K^{j} \cup E^{j}\right): D_{n} M_{b, \Omega} f(x)-D_{n} M_{b, \Omega} f_{j}(x) \geq \epsilon\right\}\right| \\
& \leq\left|\left\{x \in B^{-} \backslash\left(K^{j} \cup E^{j}\right): J_{j}(x)+\mathcal{A}_{x, b, f-f_{j}}\left(r_{j}\right) \geq \epsilon\right\}\right| \rightarrow 0 \text { as } j \rightarrow \infty .
\end{aligned}
$$

The inequality (3.116) together with the arguments similar to those in getting (3.111) yields that

$$
\left\|D_{n} M_{b, \Omega} f-D_{n} M_{b, \Omega} f_{j}\right\|_{p, E^{-} \backslash\left(K^{j} \cup E^{j}\right)} \rightarrow 0 \text { as } j \rightarrow \infty .
$$

Similar arguments to those in getting (3.113) lead to

$$
\left\|D_{n} M_{b, \Omega} f_{j}-D_{n} M_{b, \Omega} f\right\|_{p, E^{-} \cap K^{j}} \rightarrow 0 \text { as } j \rightarrow \infty .
$$

Combining (3.103) with (3.117) and (3.118) implies (3.105). This finishes the proof of the continuity part in Theorem 1.2 .

\section{Boundary values of the commutators of local Hardy-Littlewood maximal function}

We have shown the boundedness for the commutators of local Hardy-Littlewood maximal function on the Sobolev spaces, the aim of this section is to prove that the commutators of local Hardy-Littlewood maximal function preserve the zero boundary values in Sobolev's sense. Recall that $W_{0}^{1, p}(\Omega)$ denotes the Sobolev space defined as the completion of $\mathcal{C}_{0}^{\infty}(\Omega)$ with respect to the Sobolev norm. In 1998, Kinnunen and Lindqvist [17] first established that the map $M_{\Omega}: W_{0}^{1, p}(\Omega) \rightarrow W_{0}^{1, p}(\Omega)$ is bounded for all $1<p<\infty$. In this section we shall establish the following results:

Theorem 4.1. Let $1<p_{1}, p_{2}, p<\infty$ and $1 / p=1 / p_{1}+1 / p_{2}$. If $b \in W^{1, p_{2}}(\Omega)$, then the map $\left[b, M_{\Omega}\right]: W_{0}^{1, p_{1}}(\Omega) \rightarrow W_{0}^{1, p}(\Omega)$ is bounded.

Theorem 4.2. Let $1<p_{1}, p_{2}, p<\infty$ and $1 / p=1 / p_{1}+1 / p_{2}$. If $|\Omega|<\infty$ and $b \in W^{1, p_{2}}(\Omega)$, then the map $M_{b, \Omega}: W_{0}^{1, p_{1}}(\Omega) \rightarrow W_{0}^{1, p}(\Omega)$ is bounded.

The following is a Hardy-type condition for functions in $W_{0}^{1, p}(\Omega)$, which plays a key role in the proofs of Theorems 4.1 and 4.2.

Lemma 4.3. [18] Let $\Omega \subset \mathbb{R}^{n}, \Omega \neq \mathbb{R}^{n}$, be an open set. If $f \in W^{1, p}(\Omega)$ and

$$
\int_{\Omega}\left(\frac{|f(x)|}{\operatorname{dist}\left(x, \Omega^{c}\right)}\right)^{p} d x<\infty .
$$

Then $f \in W_{0}^{1, p}(\Omega)$.

Proof of Theorem 4.1. Let $f \in W_{0}^{1, p_{1}}(\Omega)$ for some $p_{1} \in(1, \infty)$ and $\left\{\varphi_{j}\right\}_{j=1}^{\infty}$ be a sequence of functions in $\mathcal{C}_{0}^{\infty}(\Omega)$ such that $\varphi_{j} \rightarrow f$ in $W^{1, p_{1}}(\Omega)$. By Theorem 1.1 we have that $\left[b, M_{\Omega}\right]\left(\varphi_{j}\right) \in W^{1, p}(\Omega)$. One can easily check that $M_{\Omega} \varphi_{j}(x)=0$ and $M_{\Omega}\left(b \varphi_{j}\right)(x)=0$ whenever $\operatorname{dist}\left(x, \Omega^{c}\right)<\frac{1}{2} \operatorname{dist}\left(\operatorname{supp} \varphi_{j}, \Omega^{c}\right)$. It follows that 
$\left[b, M_{\Omega}\right]\left(\varphi_{j}\right)(x)=0$ whenever $\operatorname{dist}\left(x, \Omega^{c}\right)<\frac{1}{2} \operatorname{dist}\left(\operatorname{supp} \varphi_{j}, \Omega^{c}\right)$. These together with (1.2) and Hölder's inequality imply that

$$
\begin{aligned}
\int_{\Omega}\left(\frac{\left[b, M_{\Omega}\right]\left(\varphi_{j}\right)(x)}{\operatorname{dist}\left(x, \Omega^{c}\right)}\right)^{p} d x & \leq\left(\frac{1}{2} \operatorname{dist}\left(\operatorname{supp} \varphi_{j}, \Omega^{c}\right)\right)^{-p}\left\|\left[b, M_{\Omega}\right]\left(\varphi_{j}\right)\right\|_{p, \Omega}^{p} \\
& \leq C_{p_{1}, p_{2}, n}\left(\|b\|_{p_{2}, \Omega}\left\|\varphi_{j}\right\|_{p_{1}, \Omega}\right)^{p}<\infty
\end{aligned}
$$

which together with $\left[b, M_{\Omega}\right]\left(\varphi_{j}\right) \in W^{1, p}(\Omega)$ leads to $\left[b, M_{\Omega}\right]\left(\varphi_{j}\right) \in W_{0}^{1, p}(\Omega)$. On the other hand, by (1.4), we have that $\left[b, M_{\Omega}\right]\left(\varphi_{j}\right) \rightarrow\left[b, M_{\Omega}\right](f)$ in $L^{p}(\Omega)$ as $j \rightarrow \infty$. By Theorem 1.1, we have

$$
\left\|\left[b, M_{\Omega}\right]\left(\varphi_{j}\right)\right\|_{p, \Omega} \leq C_{p_{1}, p_{2}, n}\|b\|_{1, p_{2}, \Omega}\left\|\varphi_{j}\right\|_{1, p_{1}, \Omega},
$$

which yields that $\left\{\left[b, M_{\Omega}\right]\left(\varphi_{j}\right)\right\}_{j=1}^{\infty}$ is a bounded sequence in $W_{0}^{1, p}(\Omega)$. A weak compactness argument implies $\left[b, M_{\Omega}\right](f) \in W_{0}^{1, p}(\Omega)$.

Proof of Theorem 4.2. By Theorem 1.2 and the arguments similar to those used in deriving Theorem 4.1, we can get the conclusion of Theorem 4.2. The details are omitted.

Acknowledgement. The authors want to express their sincerely thanks to the referees for their valuable remarks and suggestions, which made this paper more readable.

\section{References}

[1] Agcayazi, M., A. Gogatishvili, K. Koca, and R. Mustafayev: A note on maximal commutators and commutators of maximal functions. - J. Math. Soc. Japan 67:2, 2015, 581593.

[2] Aldaz, J. M., and J. PÉrez LÁzaro: Functions of bounded variation, the derivative of the one dimensional maximal function, and applications to inequalities. - Trans. Amer. Math. Soc. 359:5, 2007, 2443-2461.

[3] Bastero, J., M. Milman, and F. J. Ruiz: Commutators of for the maximal and sharp functions. - Proc. Amer. Math. Soc. 128:11, 2000, 3329-3334.

[4] Bonami, A., T. Iwaniec, P. Jones, and M. Zinsmeister: On the product of functions in BMO and $H^{1}$. - Ann. Inst. Fourier (Grenoble) 57:5, 2007, 1405-1439.

[5] Carneiro, E.: Regularity of maximal operators: recent progress and some open problems, https://arxiv.org/abs/191 2.04625 .

[6] Carneiro, E., and J. Madrid: Derivative bounds for fractional maximal functions. - Trans. Amer. Math. Soc. 369:6, 2017, 4063-4092.

[7] Carneiro, E., J. Madrid, and L. B. Pierce: Endpoint Sobolev and BV continuity for maximal operators. - J. Funct. Anal. 273:10, 2017, 3262-3294.

[8] Carneiro, E., and D. Moreira: On the regularity of maximal operators. - Proc. Amer. Math. Soc. 136:12, 2008, 4395-4404.

[9] Carneiro, E., and B. F. Svaiter: On the variation of maximal operators of convolution type. - J. Funct. Anal. 265, 2013, 837-865.

[10] García-Cuerva, J., E. Harboure, C. Segovia, and J. L. Torrea: Weighted norm inequalities for commutators of strongly singular integrals. - Indiana Univ. Math. J. 40, 1991, $1397-1420$.

[11] Gilbarg, D., and N.S. Trudinger: Elliptic partial differential equations of second order. 2nd edition. - Springer-Verlag, Berlin, 1983. 
[12] HajŁasz, P., and J. Onninen: On boundedness of maximal functions in Sobolev spaces. Ann. Acad. Sci. Fenn. Math. 29:1, 2004, 167-176.

[13] Hart, J., F. Liu, and Q. Xue: Regularity and continuity of local multilinear maximal type operators. - J. Geom. Anal. 31:4, 2021, 3405-3454.

[14] Heikkinen, T., J. Kinnunen, J. Korvenpä̈̈, and H. Tuominen: Regularity of the local fractional maximal function. - Ark. Mat. 53:1, 2015, 127-154.

[15] Hu, G., and D. YANG: Maximal commutators of BMO functions and singular integral operators with nonsmooth kernels on spaces of homogeneous type, - J. Math. Anal. Appl. 354, 2009, 249-262.

[16] Kinnunen, J.: The Hardy-Littlewood maximal function of a Sobolev function. - Israel J. Math. 100, 1997, 117-124.

[17] Kinnunen, J., and P. Lindqvist: The derivative of the maximal function. - J. Reine Angew. Math. 503, 1998, 161-167.

[18] Kinnunen, J., and O. Martio: Hardy's inequalities for Sobolev functions. - Math. Res. Lett. 4:4, 1997, 489-500.

[19] Kinnunen, J., and E. Saksman: Regularity of the fractional maximal function. - Bull. London Math. Soc. 35:4, 2003, 529-535.

[20] KorRY, S.: Boundedness of Hardy-Littlewood maximal operator in the framework of LizorkinTriebel spaces. - Rev. Mat. Complut. 15:2, 2002, 401-416.

[21] LiU, F., and H. WU: On the regularity of the multisublinear maximal functions. - Canad. Math. Bull. 58:4, 2015, 808-817.

[22] LiU, F., and H. WU: On the regularity of maximal operators supported by submanifolds. - J. Math. Anal. Appl. 453, 2017, 144-158.

[23] Liu, F., Q. Xue, and K. Yabuta: Regularity and continuity of the multilinear strong maximal operators. - J. Math. Pure Appl. 138, 2020, 204-241.

[24] Liu, F., Q. Xue, and P. Zhang: Regularity and continuity of commutators of the HardyLittlewood maximal function. - Math. Nachr. 293:3, 2020, 491-509.

[25] Luiro, H.: Continuity of the maximal operator in Sobolev spaces. - Proc. Amer. Math. Soc. $135: 1,2007,243-251$.

[26] Luiro, H.: On the regularity of the Hardy-Littlewood maximal operator on subdomains of $\mathbb{R}^{n}$. - Proc. Edinburgh Math. Soc. 53:1, 2010, 211-237.

[27] Milman, M., and T. Schonbek: Second order estimates in interpolation theory and applications. - Proc. Amer. Math. Soc. 110:4, 1990, 961-969.

[28] Zhang, P.: Characterization of Lipschitz spaces via commutators of the Hardy-Littlewood maximal function. - C. R. Acad. Sci. Paris Ser. I 355:3, 2017, 336-344.

Received 2 August 2020 • Accepted 16 April 2021 • Published online 31 December 2021

Feng Liu

Shandong University of Science and Technology

College of Mathematics and System Science

Qingdao, Shandong 266590, P. R. China

FLiu@sdust.edu.cn

Kôzô Yabuta

Kwansei Gakuin University

Research Center for Mathematics and Data Science

Gakuen 2-1, Sanda 669-1337, Japan

kyabuta3@kwansei.ac.jp

\author{
Qingying Xue \\ Beijing Normal University \\ School of Mathematical Sciences \\ Beijing 100875, P. R. China \\ qyxue@bnu.edu.cn
}

